ARTICLE

Received 6 Nov 2013 | Accepted 11 Apr 2014 | Published 23 May $2014 \quad$ DOl: 10.1038/ncomms4880

\title{
Epidermal cells help coordinate leukocyte migration during inflammation through fatty acid-fuelled matrix metalloproteinase production
}

\author{
Christopher J. Hall', Rachel H. Boyle ${ }^{1}$, Xueying Sun ${ }^{1}$, Sophie M. Wicker', June P. Misa', Geoffrey W. Krissansen', \\ Cristin G. Print ${ }^{1}$, Kathryn E. Crosier ${ }^{1} \&$ Philip S. Crosier ${ }^{1}$
}

In addition to satisfying the metabolic demands of cells, mitochondrial metabolism helps regulate immune cell function. To date, such cell-intrinsic metabolic-immunologic cross-talk has only been described operating in cells of the immune system. Here we show that epidermal cells utilize fatty acid $\beta$-oxidation to fuel their contribution to the immune response during cutaneous inflammation. By live imaging metabolic and immunological processes within intact zebrafish embryos during cutaneous inflammation, we uncover a mechanism where elevated $\beta$-oxidation-fuelled mitochondria-derived reactive oxygen species within epidermal cells helps guide matrix metalloproteinase-driven leukocyte recruitment. This mechanism requires the activity of a zebrafish homologue of the mammalian mitochondrial enzyme, Immunoresponsive gene 1. This study describes the first example of metabolic reprogramming operating within a non-immune cell type to help control its contribution to the immune response. Targeting of this metabolic-immunologic interface within keratinocytes may prove useful in treating inflammatory dermatoses.

\footnotetext{
${ }^{1}$ Department of Molecular Medicine and Pathology, School of Medical Sciences, University of Auckland, Auckland 1023, New Zealand. Correspondence and requests for materials should be addressed to P.S.C. (email: ps.crosier@auckland.ac.nz).
} 
nvestigating how metabolism shapes the immune response is gaining significant attention due to the emerging connection between metabolic and inflammatory processes. Fuelled by the obesity epidemic, this new field of immunometabolism is underpinned by observations that inflammation is a hallmark of many chronic metabolic conditions ${ }^{1,2}$. In addition to the activation of immune cells within metabolic tissues, functional heterogeneity of immune cells can be regulated, on many levels, by cell-intrinsic metabolic reprogramming ${ }^{3-5}$. Whether nonimmune cells also contribute to the immune response through coupling metabolism and immune function is, at present, unclear.

Mitochondria are emerging as key organizers of innate immune cell function ${ }^{6}$. One mechanism through which mitochondria can influence immune cell activity is through elevated production of mitochondrial reactive oxygen species (mROS). Although a major source of cellular ROS, mROS have traditionally been regarded as insignificant by-products of oxidative phosphorylation (OXPHOS). More recent studies have identified inflammation-responsive mechanisms that elevate mROS and shown that mROS can have direct antibacterial activity and influence the expression of immuneresponse genes ${ }^{6-8}$. Whether non-immune cells also couple metabolic and inflammatory processes during their contribution to the immune response is unclear.

In addition to providing a physical barrier protecting the host from its environment, the skin is a critical component of the immune system ${ }^{9}$. The immune response during cutaneous inflammation relies on finely tuned communication between immune cell subsets and non-immune cells such as epidermal keratinocytes ${ }^{10}$. Dysregulation of this communication can lead to inadequate or persistent inflammation that characterizes many inflammatory dermatoses ${ }^{11}$. In response to infection or injury keratinocytes produce matrix metalloproteinases (MMPs), a family of enzymes that collectively degrade all components of the extracellular matrix $(\mathrm{ECM})^{12,13}$. The recruitment of leukocytes from the bloodstream and their migration through the ECM to sites of inflammation is, in part, dependent upon MMP activity. It has been suggested that keratinocyte-derived MMP9 production facilitates migration of leukocytes into the inflamed epidermis during cutaneous inflammation ${ }^{13}$. Both the expression and activity of MMPs has been shown to be directly regulated by $\mathrm{mROS}^{14}$. Furthermore, tumour necrosis factor alpha (TNF- $\alpha$ )-driven MMP9 expression within human keratinocytes is ROS-dependent ${ }^{15}$. Collectively, these data suggest mROS production within keratinocytes may promote leukocyte migration through regulation of MMP activity. These results also imply a possible connection between the metabolic process of OXPHOS within epidermal cells and leukocyte recruitment.

The zebrafish is a well-established model to investigate innate immune cell function ${ }^{16}$. By 2 days post fertilization (dpf) zebrafish larvae are populated with neutrophil and macrophage lineages. Live imaging these innate immune cells within transgenic reporter lines has revealed remarkable conservation of function with their mammalian equivalents ${ }^{17-20}$. Live imaging analyses have also given unique insights into leukocyte behaviour during wound-associated cutaneous inflammation ${ }^{21-24}$. In contrast to the mammalian epidermis that consists of proliferating and differentiated keratinocytes within several strata, the epidermis of embryonic/larval zebrafish is relatively simple consisting of an outermost enveloping and inner epidermal basal layer ${ }^{25,26}$. Despite this simple architecture, functional similarities exist between the zebrafish and mammalian epidermis, supporting the use of zebrafish as a model of skin diseases ${ }^{26-28}$.

Exploiting the live imaging potential of the zebrafish system, previous work in our group has revealed activated macrophages utilize $\beta$-oxidation of fatty acids to fuel OXPHOS-coupled mROS that contributes to macrophage effector function ${ }^{7}$. This metabolic reprogramming of macrophages requires a mitochondrial enzyme encoded by immunoresponsive gene 1 (irg1), a vertebrate homologue of a bacterial enzyme necessary for fatty acid metabolism ${ }^{29}$. Zebrafish possess 2 IRG1 homologues, irg1 and irg1-like (irg1l), most likely the result of a duplication event. A recent study has suggested a function for Irgll during cutaneous inflammation ${ }^{30}$. We predicted that Irg1l may couple metabolic and immunological processes, similar to its paralog Irg1. Using the zebrafish system to explore the function of Irg1l, we uncover a new Irg1l-dependent signalling pathway that connects metabolic and inflammatory processes within epidermal cells during inflammation. We demonstrate expression of irgll within epidermal cells in response to live bacterial infection and wound-induced inflammation, where it contributes to leukocyte recruitment. In response to bacterial infection, epidermal cells enhance their production of mROS in a process dependent on Irg1l and fuelled by fatty acid $\beta$-oxidation. We further show that Irg1l and $\beta$-oxidation are necessary for infection- and wound-responsive $m m p 9$ expression within epidermal cells that demonstrate elevated mitochondrial uptake of fatty acids. Finally, we demonstrate elevated abundance of IRG1 within mitochondria of keratinocytes in a mouse model of cutanous inflammation (atopic dermatitis) supporting the translation of our zebrafish findings to a mammalian setting. We propose a new mechanism where Irg1l-dependent $\beta$-oxidation-fuelled mROS production within epidermal cells contributes to MMP-9-driven leukocyte migration during inflammation. For the first time, this study connects inflammation-driven changes in epidermal cell metabolism with their contribution to the immune response. This suggests that metabolic reprogramming, as a mechanism to help orchestrate the immune response, is not restricted to immune cells.

\section{Results}

irg1l is expressed by epidermal cells following infection. We first identified zebrafish Irg1l (a homologue of mammalian IRG1) as a gene upregulated in response to live Salmonella enterica serovar Typhimurium (hereafter referred to as Salmonella) infection using the GeneChip Zebrafish Genome Array (Affymetrix) (Fig. 1a), consistent with a previous study ${ }^{30}$. Quantitative (q) PCR analysis of irgll expression following Salmonella infection ( $\sim 600$ colony-forming units (cfu) injected into the hindbrain ventricle of $2 \mathrm{dpf}$ larvae) revealed elevated expression from $1 \mathrm{~h}$ post injection (hpi), when compared with PBS-injected controls (Fig. 1b). Infection-responsive expression of irgll peaked at 1 day post injection (dpi) before returning to 'steady-state' levels by 3 dpi (Fig. 1b). Throughout this study, unless otherwise stated, an infection dose of $600 \mathrm{cfu}$ of Salmonella was injected into the hindbrain ventricle of $2 \mathrm{dpf}$ larvae to stimulate an antibacterial immune response. When compared with PBSinjected controls, Salmonella-injected larvae (2 hpi) possessed infection-responsive irgll expression within cells covering the infection site as well as within nasal and neuromast epithelial cells (Fig. 1c,d). Constitutive expression of irg1l was detected within epithelial cells of the oropharyngeal region and cloaca (Fig. 1c). Injection of $E$. coli lipopolysaccharide (LPS) also stimulated similar early irg1l expression (Supplementary Fig. 1). Of note, LPS-stimulated irgll expression did not persist as long as that induced by live Salmonella injection (Supplementary Fig. 1). By 1 $\mathrm{dpi}$, infection-responsive expression of irgll became largely restricted to neuromasts of the developing lateral line system (Supplementary Fig. 2a,b). Some infected larvae also possessed irg1l expression within the intestine and pronephric tubules 
a
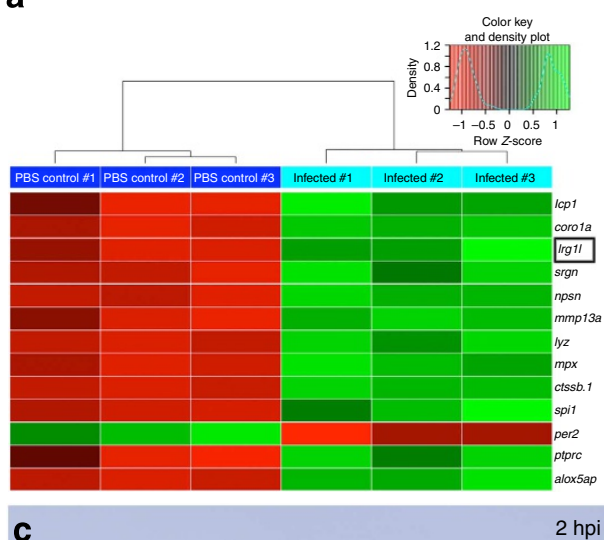

c
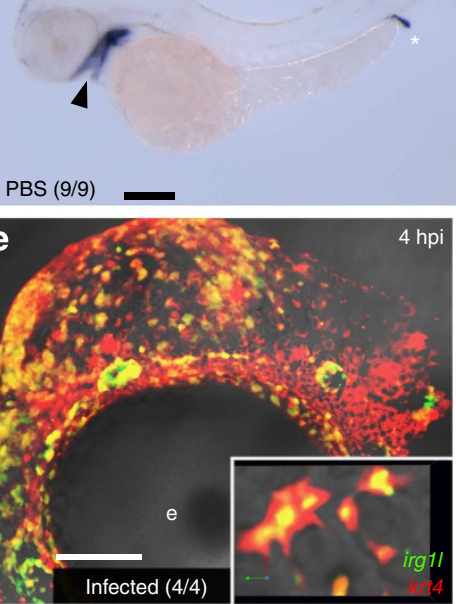

b

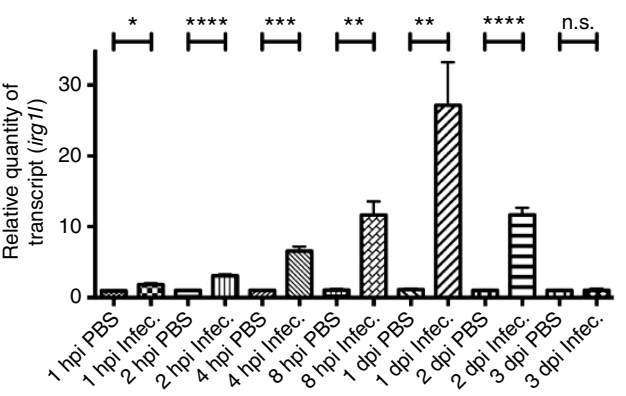

pi

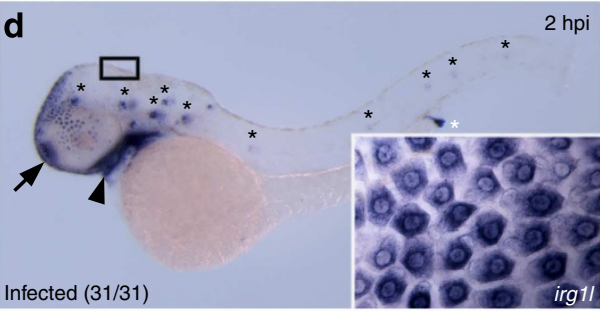

irg1I Infected (31/31)

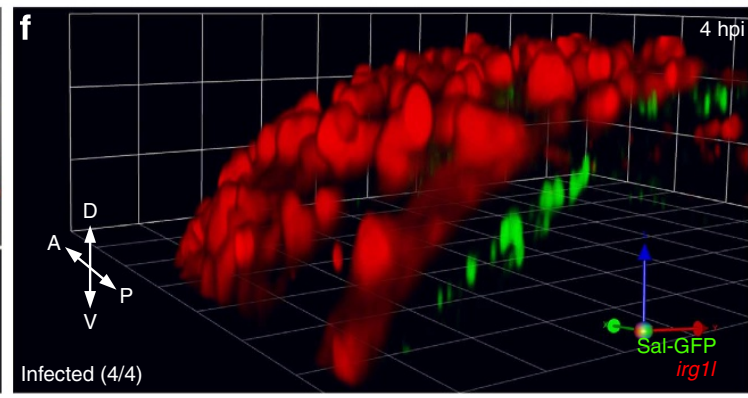

Figure 1 | Irg11 is expressed within krt4 ${ }^{+}$epidermal cells following Salmonella infection. (a) Heat map illustrating differentially abundant mRNAs between infected (hindbrain Salmonella-injected) and PBS-injected larvae (in biological triplicate). (b) qPCR analysis of irg1l expression following infection, measured at 1 hpi, 2 hpi, 4 hpi, 8 hpi, 1 dpi, 2 dpi and 3 dpi, relative to PBS-injected controls (mean \pm s.d.; $n=3$ biological replicates, $\sim 50$ larvae per group). $(\mathbf{c}, \mathbf{d})$ Expression analysis of irg 11 within PBS control and infected larvae at $2 \mathrm{hpi}$, respectively $(n=2$ separate infection experiments, lateral views, anterior to left). Inset, magnified dorsal view of boxed region in d. Arrowhead and white asterisk mark constitutive irg 11 expression within oropharyngeal region and cloaca, respectively. Arrow and black asterisks mark infection-responsive irgll expression within nasal and neuromast epithelium, respectively.

(e) Expression of irgll within krt4 ${ }^{+}$epidermal cells covering the head following infection, as detected at 4 hpi (lateral view, anterior to left). Inset, magnified view of individual cells co-expressing irgll and krt4. (f) 3D transverse view demonstrating irgll expression within epidermal cells of the midbrain/hindbrain region overlying injected GFP-expressing Salmonella, at 4 hpi. Numbers represent frequencies of larvae with displayed phenotypes. Scale bars, $100 \mu \mathrm{m}$ in c; $50 \mu \mathrm{m}$ in e. Statistical significance determined using a Student's $t$-test. ${ }^{\star} P$-value $<0.05$; ${ }^{\star \star} P$-value $<0.01$; ${ }^{\star \star \star} P$-value $<0.001$; ${ }^{\star \star \star \star} P$-value $<0.0001$; n.s., not significant; e, eye; $A$, anterior; $P$, posterior; $D$, dorsal; $V$, ventral.

(Supplementary Fig. 2c,d). In light of the well-recognized contribution that the epidermis/skin provides to a functional immune system, we investigated the role of Irg1l within the epidermis. Expression analysis revealed that epithelial cells with infection-responsive irgll expression, surrounding the infection site, were also positive for the epidermal marker keratin 4 (krt4), as detected at $4 \mathrm{hpi}$ (Fig. 1e). To visualize epidermal irgll expression with respect to injected bacteria, we utilized GFPexpressing Salmonella (hereafter referred to as Sal-GFP) ${ }^{31}$. Injected Sal-GFP were observed in close proximity to (but never within) overlying $\operatorname{irg}^{+} \mathrm{l}^{+}$epidermal cells (Fig. 1f). Transcriptome profiling has previously demonstrated expression of irgll to be elevated in response to both intravenous injection of, and immersion in, the natural fish pathogen Edwardsiella tard ${ }^{30}$. In contrast to this study, we were not able to detect epidermal expression of irgll following immersion in live Salmonella or LPS, as detected by whole-mount in situ hybridization (WMISH) analysis (Supplementary Fig. 2e-h). This suggests, in our infection model, host-derived endogenous signals contribute to infection-responsive expression of $i r g 1 l$ within epidermal cells.

These results demonstrate that $i r g 1 l$ is rapidly expressed within epidermal cells in response to bacterial infection.

Macrophages are required for epidermal irgll expression. We have previously shown macrophages respond, and functionally contribute, to the immune response in our hindbrain infection model $^{31}$. We next investigated whether host-derived signals necessary for epidermal irg1l expression were macrophagedependent.

We took advantage of the ability of spleen focus-forming virus proviral integration oncogene (Spi1)- and granulocyte colony stimulating factor receptor (Gcsfr)-targeting morpholino oligonucleotides (MOs) to deplete larvae of both the macrophage and neutrophil lineages, to first assess the myeloid-lineage contribution to infection-responsive epidermal irg1l expression ${ }^{31,32}$. 


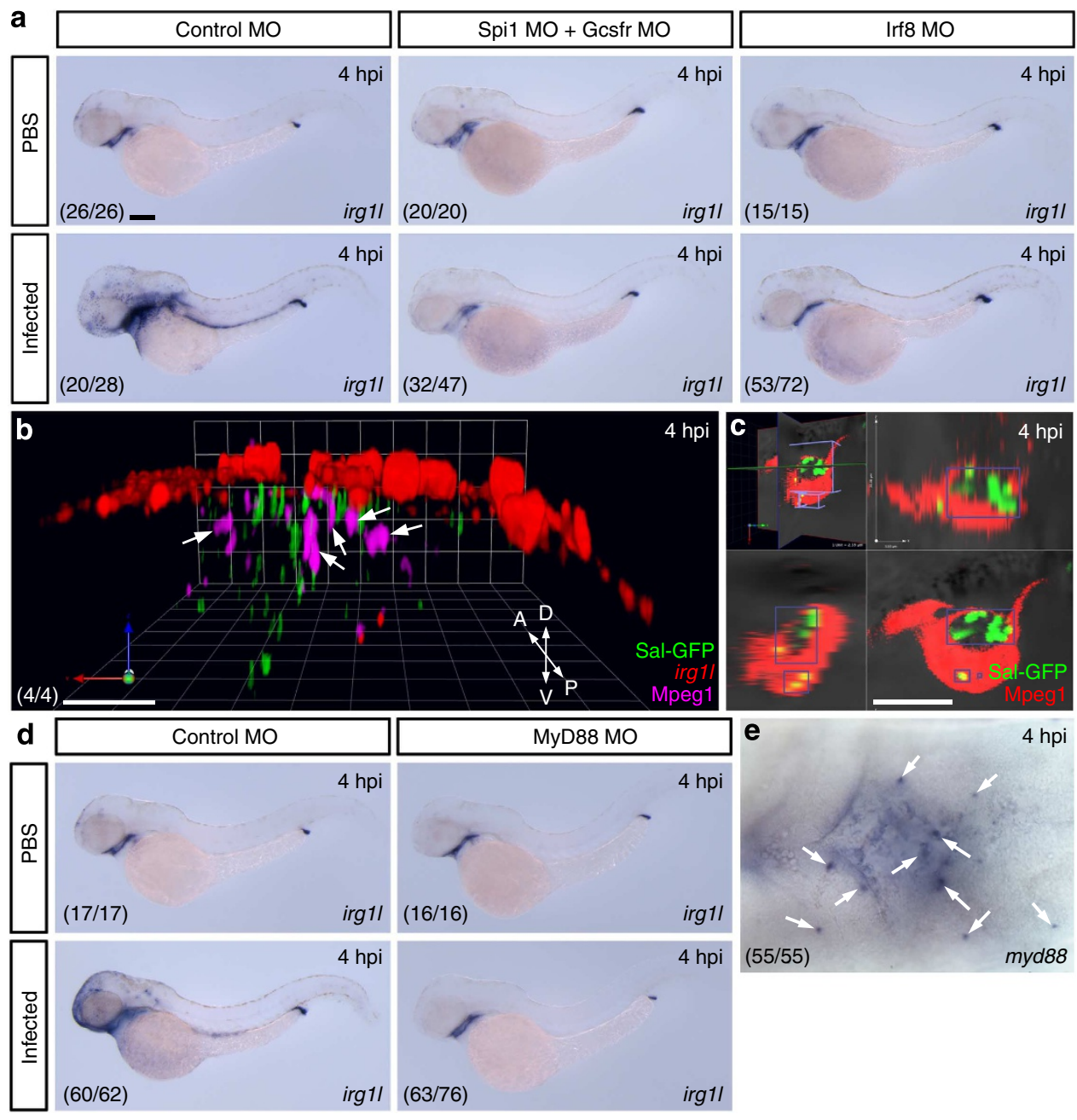

Figure 2 | Infection-responsive epidermal expression of irg1l is dependent upon macrophages and Myd88. (a) Expression of irg1/ within control MO-, Spi1 MO + Gcsfr MO- and Irf8 MO-injected larvae following PBS and Salmonella injection, as detected at 4 hpi $(n=2$ separate infection experiments, lateral views, anterior to left). Remainder of infected control MO-injected (8/28), Spi1/Gcsfr- (15/47) and Irf8- (19/72) depleted larvae displayed weak epidermal irg1/ expression. (b) 3D transverse view of midbrain/hindbrain region of $\mathrm{Tg}$ (mpeg1:Gal4/UAS:Kaede), larvae demonstrating irg7l-expressing epidermal cells overlying Sal-GFP and Mpeg1+ macrophages (white arrows) following infection, as detected by immunofluorescence at 4 hpi. (c) Live imaging Sal-GFP within Mpeg1+ macrophages, at 4 hpi, within midbrain/hindbrain region of $\mathrm{Tg}$ (mpeg1:mCherry) larvae following infection. (d) Expression of irgll within control MO-injected and MyD88-depleted larvae following PBS and Salmonella injection, as detected at 4 hpi $(n=3$ separate infection experiments, lateral views, anterior to left). Remainder of infected control MO-injected (2/62) and MyD88-depleted (13/76) larvae displayed weak epidermal irgll expression. (e) Expression of myd88 within the midbrain/hindbrain region, at 4 hpi, following infection $(n=3$ separate infection experiments, dorsal view, anterior to left). Arrows mark presumptive myd88-expressing macrophages. Numbers represent frequencies of larvae with displayed phenotypes. Scale bars, $100 \mu \mathrm{m}$ in $\mathbf{a} ; 50 \mu \mathrm{m}$ in $\mathbf{b} ; 10 \mu \mathrm{m}$ in c. A, anterior; P, posterior; $\mathrm{D}$, dorsal; $V$, ventral.

Larvae depleted of both Spil and Gcsfr demonstrated a decrease in epidermal irg1l expression following infection (Fig. 2a). To differentiate whether this myeloid-lineage contribution to infection-responsive irg1l expression was at the level of the macrophage or neutrophil, we next evaluated irgll expression within interferon regulatory factor 8 (Irf8)-depleted larvae following infection. The transcription factor Irf8 has been shown to regulate macrophage versus neutrophil lineage commitment such that Irf8-depleted larvae possess an expanded neutrophil compartment and depleted numbers of macrophages ${ }^{33}$. Similar to larvae depleted of both Spi1 and Gcsfr, Irf8-depleted larvae demonstrated a decrease in epidermal irgll expression following infection (Fig. 2a). These results suggest macrophages contribute to infection-responsive epidermal irg1l expression. To visualize macrophages in relation to injected bacteria and epidermal irg1l expression, we exploited the macrophage-lineage marking $\mathrm{Tg}$ (mpeg1:Gal4/UAS:Kaede) reporter line ${ }^{17}$. Analysis of irg1l expression within Tg (mpeg1:Gal4/UAS:Kaede) larvae following Sal-GFP injection showed macrophage expressed 1 (Mpeg1) ${ }^{+}$macrophages and bacteria in close proximity to overlying $\mathrm{irg}^{+} \mathrm{l}^{+}$epidermal cells, as assessed at 4 hpi (Fig. 2b). Live imaging infected Tg(mpeg1:mCherry $)^{17}$ larvae demonstrated that these macrophages were highly phagocytic toward the injected Sal-GFP (Fig. 2c).

A well-characterized mechanism through which innate immune cells detect bacteria is via the Toll-like receptor (TLR) class of pattern recognition receptors ${ }^{34}$. Cell surface and intracellular TLRs detect specific pathogen-associated molecular patterns, stimulating signalling cascades that couple pathogen detection to downstream immune cell functions, including cytokine production. TLR signalling is largely dependent on the adaptor molecule myeloid differentiation primary response gene 88 (MYD88). We have previously shown that innate immune 

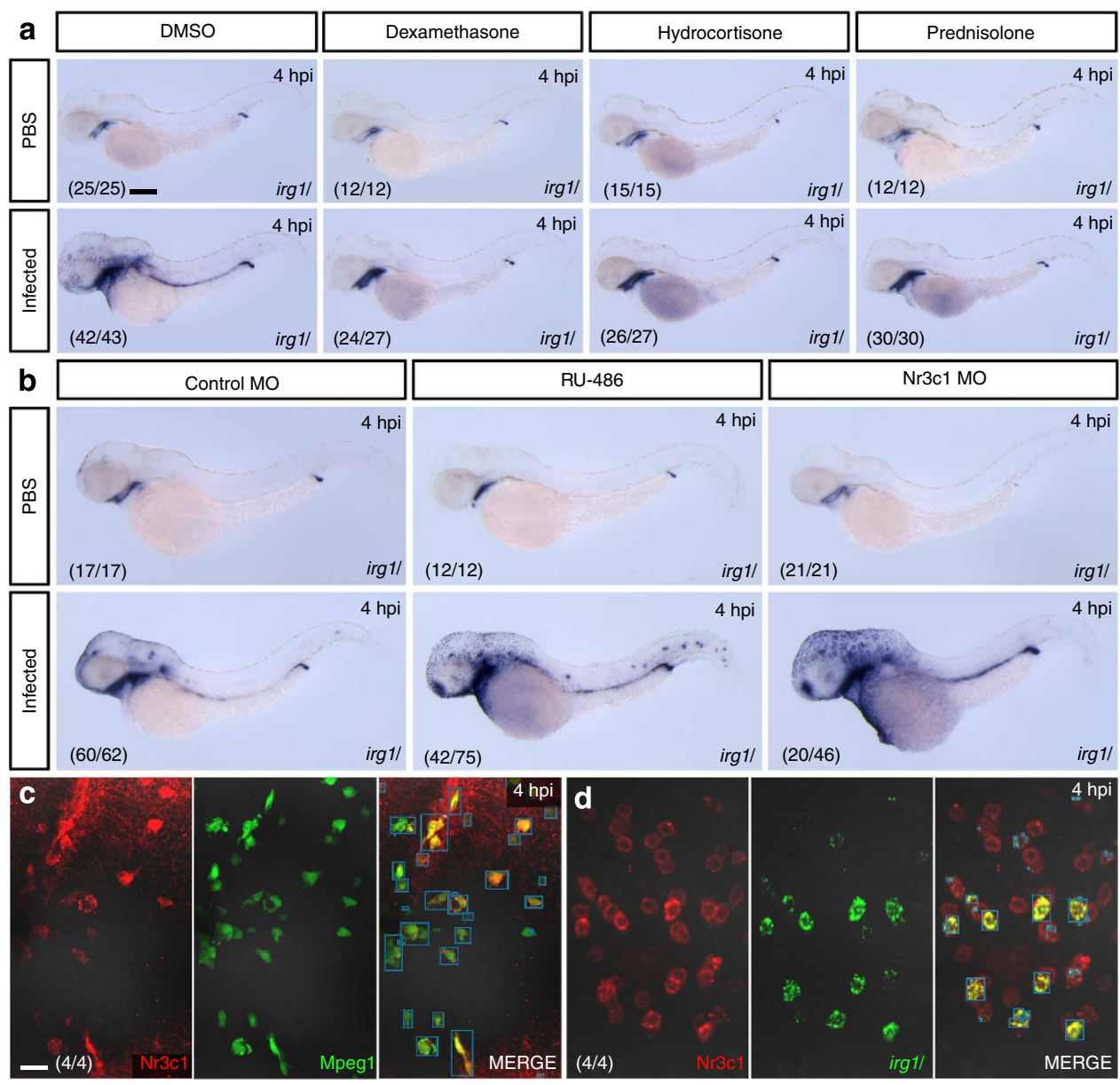

Figure 3 | GC/GR signalling suppresses infection-responsive expression of irg1/ in epidermal cells. (a) Expression of irg7/ within PBS control and infected larvae following DMSO, dexamethasone, hydrocortisone or prednisolone treatments, as detected at 4 hpi $(n=2$ separate infection experiments, lateral views, anterior to left). Remainder of infected DMSO- (1/43), dexamethasone- (3/27) and hydrocortisone-treated (1/27) larvae displayed weak epidermal irg1l expression. (b) Expression of irg1l within control MO-injected, RU-486-treated and Nr3c1-depleted larvae following PBS control and Salmonella injection, as detected at $4 \mathrm{hpi}(n=2$ separate infection experiments, lateral views, anterior to left). Remainder of infected control MO-injected larvae (2/62) possessed weak epidermal irg1l expression. Remainder of infected RU-486-treated (33/75) and Nr3c1-depleted (26/46) larvae displayed irg1l expression similar to that shown for control MO-injected/infected. (c) Immunofluorescence detection of Nr3c1 within Mpeg1 ${ }^{+}$macrophages, at 4 hpi, within the midbrain/hindbrain region of $\mathrm{Tg}$ (mpeg1:EGFP) larvae following infection. (d) Immunofluorescence detection of Nr3c1 within irg1/-expressing epidermal cells, at $4 \mathrm{hpi}$, overlying the midbrain/hindbrain infection region. Boxes mark colocalized signal. Numbers represent frequencies of larvae with displayed phenotypes. Scale bars, $100 \mu \mathrm{m}$ in $\mathbf{a} ; 20 \mu \mathrm{m}$ in c.

cells in zebrafish express TLR adaptor molecules, including myd88 (ref. 18). In addition, zebrafish MyD88 has been demonstrated to contribute to pathogen clearance following infection $^{35}$. To assess whether MyD88-dependent TLR signalling is required for irg1l expression within epidermal cells following infection, we analysed irg1l expression within infected MyD88depleted larvae. Epidermal expression of irg1l was not detected within infected MyD88-depleted larvae (Fig. 2d). Analysis of myd88 expression within infected larvae revealed expression within putative macrophages in the midbrain/hindbrain region (Fig. 2e). Of note, expression was not detected within the overlying epidermis.

These results suggest infection-responsive expression of irgll within the epidermis requires macrophage detection of bacteria through a MyD88-dependent mechanism.

GC signalling suppresses epidermal irg1l expression. Glucocorticoids (GCs) are a class of steroid hormone that largely suppress the immune response. Through binding the glucocorticoid receptor (GR), GCs upregulate anti-inflammatory, while repressing pro-inflammatory, gene expression. GCs have been shown to possess conserved anti-inflammatory activity in larval zebrafish (Supplementary Fig. $3 a-c)^{36}$. We next investigated whether the infection-responsive expression of irg1l within epidermal cells was sensitive to the anti-inflammatory activity of GC/GR signalling.

To assess the effect of GCs on epidermal irg1l expression we co-injected Salmonella with dexamethasone, hydrocortisone and prednisolone and analysed irg $1 \mathrm{l}$ expression at $4 \mathrm{hpi}$. In contrast to Salmonella alone, epidermal irg1l expression was not detected in co-injected larvae (Fig. 3a), suggesting GCs suppress infectionresponsive expression of $i r g 1 l$ within epidermal cells. To evaluate the requirement for GR signalling during GC-mediated irg1l suppression, we co-injected Salmonella with the GR antagonist RU-486 and infected larvae depleted of the zebrafish ortholog of nuclear receptor subfamily 3 , group $\mathrm{C}$, member 1 (NR3C1) (encoding the GR), then assessed irg1l expression. When compared with control MO-injected infected larvae, RU-486treated and Nr3c1-depleted larvae demonstrated elevated 

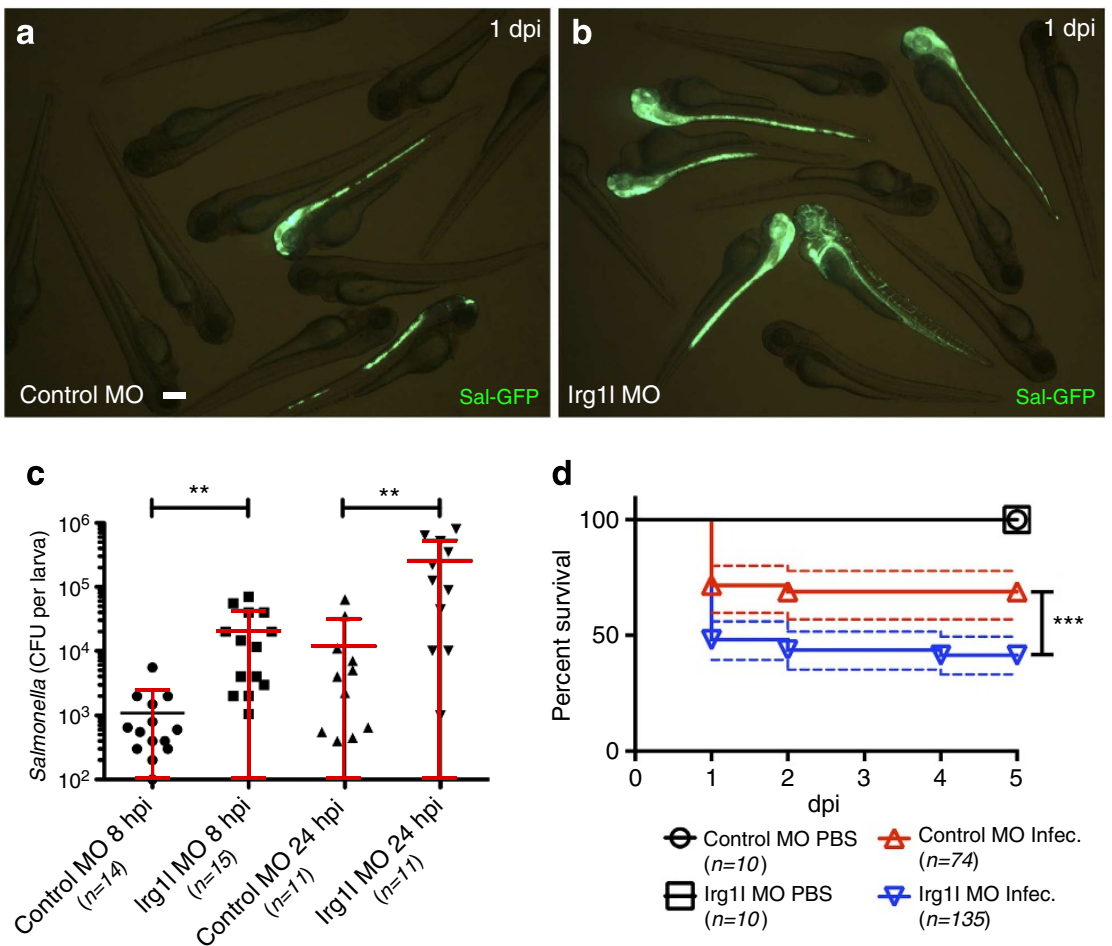

Figure 4 | Irg11-depleted larvae demonstrate diminished bacterial clearance and reduced survival following infection. (a,b) Live imaging of control MO-injected and Irg1l-depleted larvae, respectively, following infection with GFP-labelled Salmonella, imaged at 1 dpi. (c) CFU counts of remaining GFP-labelled Salmonella within individual infected control MO-injected and Irg1l-depleted larvae quantified at 8 and 24 hpi (mean \pm s.d.; $n=2$ experiments). Statistical significance determined using a Student's t-test. (d) Kaplan-Meier survival graph demonstrating \% survival of control MO-injected PBS-injected, Irg1l-depleted PBS-injected, control MO-injected infected and Irg1l-depleted infected larvae from 1 to 5 dpi. Significance of curve differences for control MO-infected and Irg1l-depleted infected groups calculated using Log-rank (Mantel-Cox) test, $n=3$ separate infection experiments ( $95 \%$ c.i. represented by dashed lines). Scale bar, $200 \mu \mathrm{m}$ in $\mathbf{a} .{ }^{\star \star} P$-value $<0.01 ;{ }^{\star \star \star} P$-value $<0.001$.

infection-responsive epidermal irg1l expression (Fig. 3b). In zebrafish, endogenous GCs are produced by the inter-renal tissue, which functions as an adrenal gland-equivalent. Inter-renal tissue and its steroidogenic activity can be ablated through knockdown of nuclear receptor subfamily 5, group A, member 1a (Nr5a1a) (Supplementary Fig. 3d,e) ${ }^{37}$. A similar increase in epidermal irgll expression following infection was observed within larvae depleted of Nr5ala (Supplementary Fig. 3f). Immunofluorescence detection of $\mathrm{Nr} 3 \mathrm{cl}$ within infected larvae revealed $\mathrm{Nr} 3 \mathrm{cl}$ protein localized to Mpeg1 ${ }^{+}$macrophages within, and irgll-expressing epidermal cells overlying, the midbrain/ hindbrain infection region (Fig. 3c,d). The greater sensitivity of this tyramide signal amplification-based fluorescence detection method revealed irg1l expression extended throughout the trunk within $\mathrm{Nr}_{3} \mathrm{cl}^{+}$epidermal cells (Supplementary Fig. 4). Of note, $\mathrm{Nr} 3 \mathrm{cl}$ also colocalized with other irgll-expressing epithelial tissues including the neuromasts, cloaca, nasal epithelium and the oropharngeal region (Supplementary Fig. 4).

Together, these results support a model where infectionresponsive epidermal expression of $i r g 1 l$ is suppressed by the antiinflammatory activity of GCs, most likely at the level of GR/ $\mathrm{Nr} 3 \mathrm{cl}^{+}$macrophages and/or epidermal cells.

Irg1l contributes to bacterial clearance. To examine whether Irg1l contributes to the clearance of injected bacteria, we monitored bacterial persistence within Irg1l-depleted larvae infected with Sal-GFP. When compared with control MO-injected larvae, Irg1-depleted larvae demonstrated a significant increase in bacterial persistence, as assessed by live fluorescence microscopy (Fig. 4a,b and Supplementary Fig. 5) and recovered bacteria (Fig. 4c). This defective bacterial clearance resulted in decreased survival post-infection (Fig. 4d). These data support a functional role for Irg1l during clearance of injected bacteria.

Irg11 contributes to epidermal $m m p 9$ expression. A feature of cutaneous inflammation associated with infection is the infiltration of innate immune cells, including monocytes/macrophages and neutrophils, into the dermis and epidermis. Central to the recruitment of leukocytes is the ECM-degrading activity of the MMP family of proteases ${ }^{13,38}$. Similar to mammals, bacterial infection can elevate the expression of $m m p 9$ within the zebrafish larval epidermis (Supplementary Fig. 6), where it contributes to macrophage recruitment ${ }^{39}$. We next investigated whether infection-responsive expression of $\mathrm{irgll}$ within the epidermis contributes towards $m m p 9$ expression and the recruitment of macrophages to the infection site.

Fluorescent expression analysis confirmed irg1l and mmp9 expression colocalized within epidermal cells following infection (Fig. 5a,b). qPCR analysis of $m m p 9$ expression within control MO-injected and Irg1l-depleted larvae at 1, 2 and 4 hpi revealed that Irg1l contributed to infection-responsive $m m p 9$ expression (Fig. 5c). This result was supported when assessing $m m p 9$ expression within infected control MO and Irg1l-depleted larvae (Fig. 5d,e). Irg1l-depleted Tg(mpeg1:EGFP) larvae also demonstrated reduced macrophage recruitment to the infection site, when compared with control MO-injected $\operatorname{Tg}($ mpeg1:EGFP) 

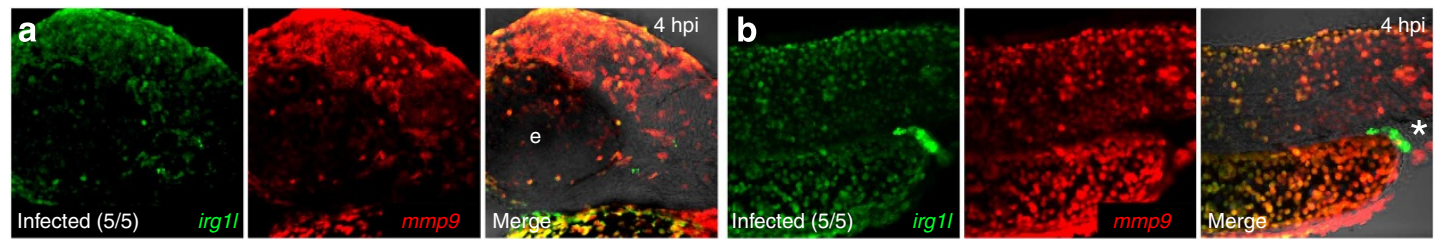

C

营

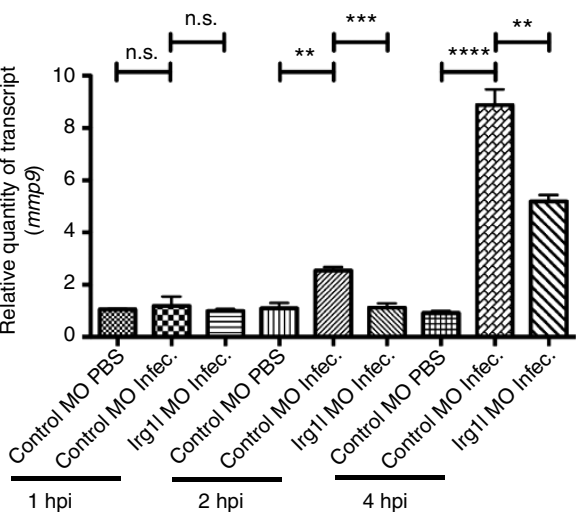

d
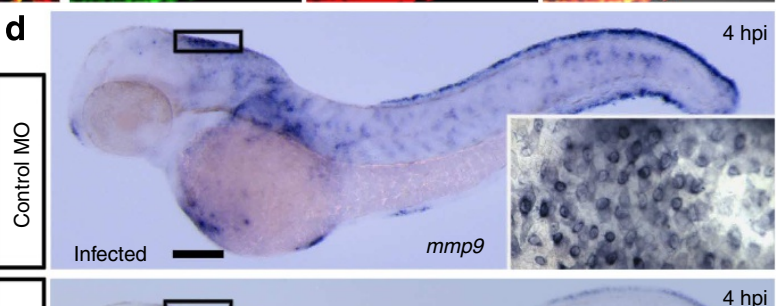

\section{.}
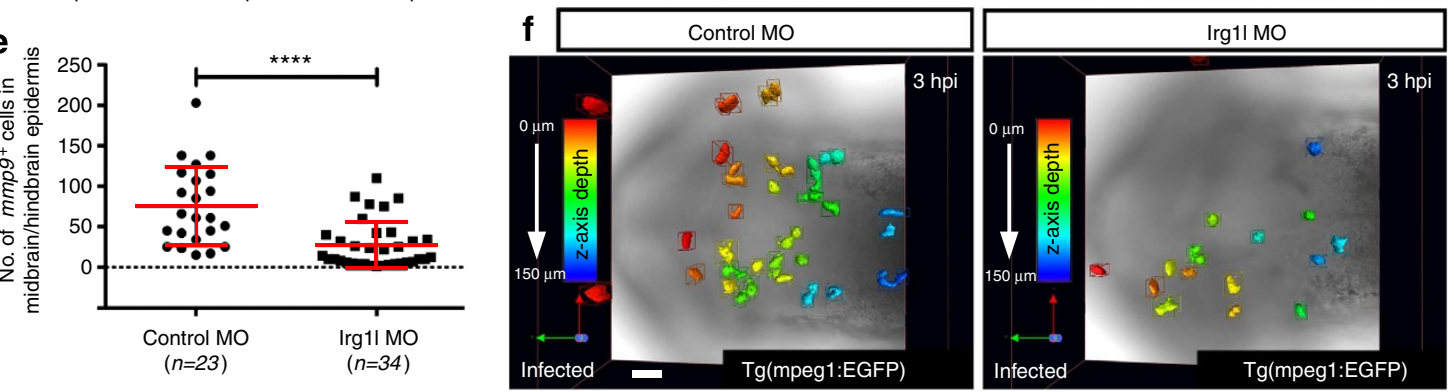

g

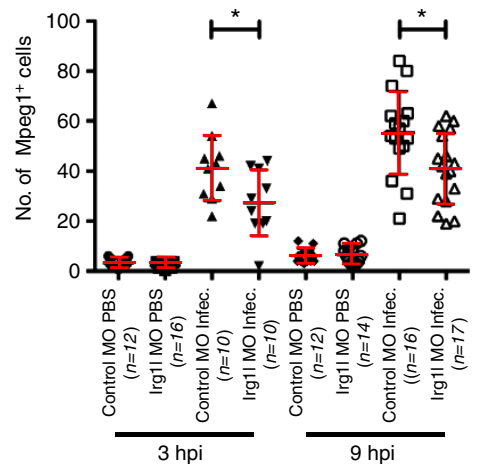

Figure 5 | Irg1l contributes to infection-responsive mmp9 expression within epidermal cells and the recruitment of macrophages to the infection site.

$(\mathbf{a}, \mathbf{b})$ Dual expression analysis of irgll and mmp9 within the head and trunk, respectively, following infection, as detected at 4 hpi (lateral views, anterior to left). White asterisk marks irg1l-expressing cloaca. (c) qPCR analysis of mmp9 expression within control MO-injected and Irg1l-depleted larvae following PBS control and Salmonella injection, detected at 1, 2 and 4 hpi (mean \pm s.d.; $n=3$ biological replicates, $\sim 50$ larvae per group). (d) Expression of mmp9 within control MO-injected and Irg1l-depleted larvae following infection, as detected at $4 \mathrm{hpi}$ (lateral views, anterior to left). Insets represent magnified dorsal views of boxed regions. (e) Quantification of $m m p 9^{+}$epidermal cells covering the midbrain/hindbrain region of individual infected larvae as shown in d (mean \pm s.d.; $n=2$ separate infection experiments). (f) Detection of Mpeg1+ cells, at 3 hpi, within the midbrain/hindbrain region of control MO-injected and Irg1l-depleted Tg(mpeg1:EGFP) larvae following infection (dorsal views, anterior to left). Individual macrophages are pseudo-colored to

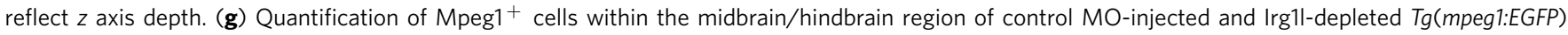
larvae (as detected in $\mathbf{f}$ ), at 3 and 9 hpi, following PBS or Salmonella injections (mean \pm s.d.; $n=2$ separate infection experiments). Scale bars, $50 \mu m$ in $\mathbf{a}$; $100 \mu \mathrm{m}$ in d; $20 \mu \mathrm{m}$ in f. Statistical significance determined using a Student's $t$-test. ${ }^{\star} P$-value $<0.05 ;{ }^{\star \star} P$-value $<0.01 ;{ }^{\star \star \star} P$-value $<0.001 ;{ }^{\star \star \star \star \star} P$-value $<0.0001$; n.s., not significant; e, eye.

larvae, despite maintaining unaltered (whole larvae) numbers of macrophages (Fig. 5f,g and Supplementary Fig. 7).

These results suggest that Irgll contributes to the recruitment of macrophages to the infection site through regulating epidermal mmp9 expression.
Irg1l regulates epidermal $m m p 9$ expression following wounding. In addition to infection, cutaneous inflammation associated with wound healing, allergy and/or trauma also requires the timely recruitment of specific leukocyte subsets. Employing a wellcharacterized tail transection model of acute wound-associated 

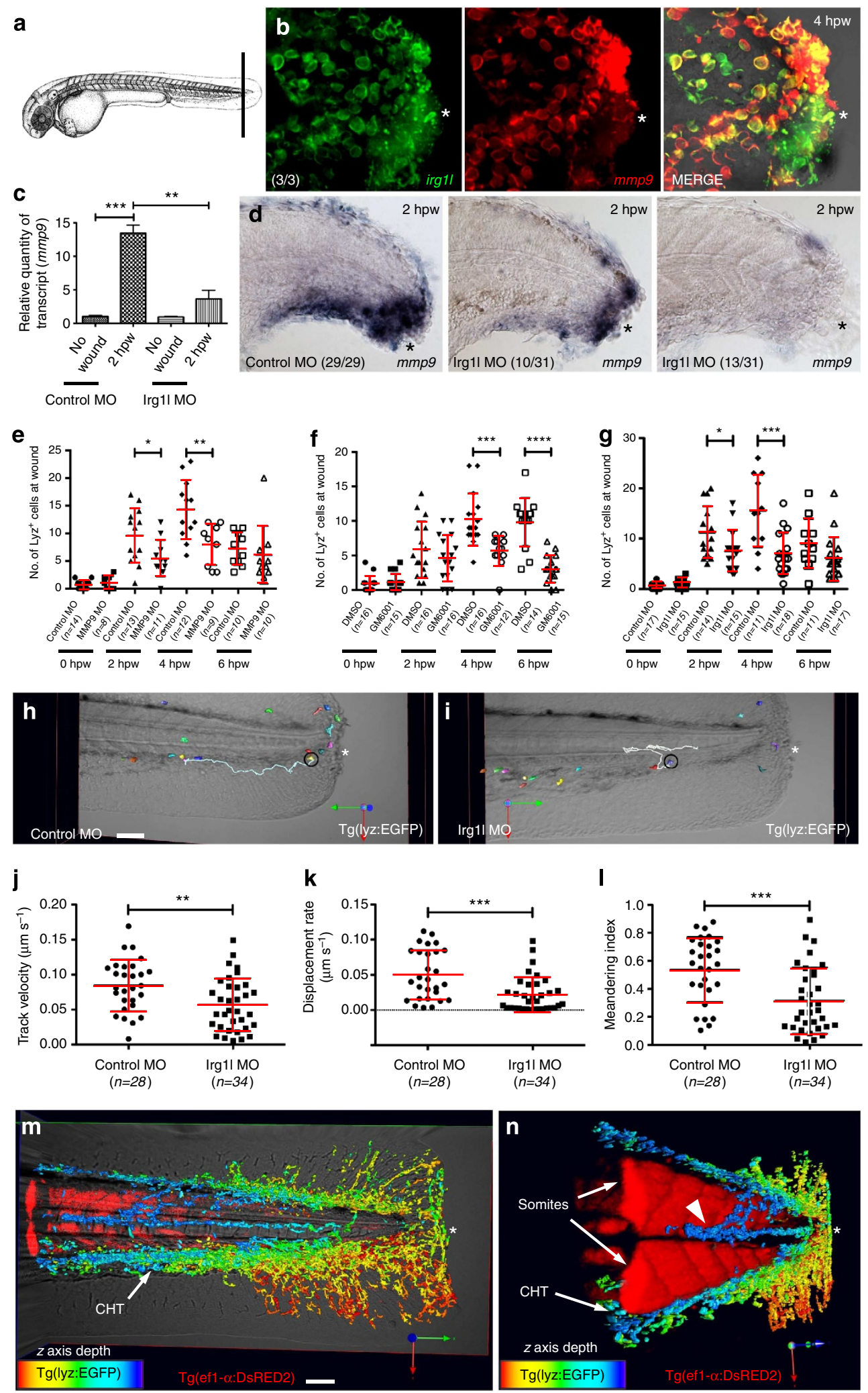

inflammation (Fig. 6a) ${ }^{17,40}$, we examined whether irgll was expressed in response to wounding and, if so, whether it helped coordinate the inflammatory phase of wound healing.

Similar to that induced by bacterial injection, irg1l was expressed within epidermal cells at the wound site by 1 hour post wounding (hpw) (Supplementary Fig. 8a,b). Expression analysis confirmed that irg1l-expressing epidermal cells also expressed $m m p 9$ (Fig. 6b). Irg1l-depleted larvae demonstrated a reduction in wound-induced epidermal $m m p 9$ expression, when compared with control larvae, suggesting Irg1l is required, at least in part, for wound-induced epidermal mmp9 expression (Fig. 6c,d). As 'first responders' during inflammation, neutrophils are the first leukocytes to arrive at wounded tissues. Live imaging of wounded transgenic zebrafish larvae possessing fluorescent 
Figure 6 | Irg1l contributes to epidermal mmp9 expression and the recruitment of neutrophils to wounded tissues. (a) Schematic of tail transection within $2 \mathrm{dpf}$ larvae (all wounds were generated at the same anatomic location). (b) Expression of irg $1 /$ and mmp9 within larvae at $4 \mathrm{~h}$ post wounding (hpw), magnified view of transected tail (lateral views, anterior to left). (c) qPCR analysis of mmp9 expression within control MO-injected and Irg1l-depleted larvae, relative to non-wounded controls, at $2 \mathrm{hpw}$ (mean \pm s.d.; $n=3$ biological replicates, $\sim 50$ larvae per group). (d) Expression of mmp 9 within control MO-injected and Irg1l-depleted larvae at $2 \mathrm{hpw}$ ( $n=2$ separate wounded cohorts, lateral views, anterior to left). Magnified views of transected tails revealing Irg11-depleted larvae with reduced (10/31) and abolished (13/31) wound-induced mmp9 expression. Remainder of wounded Irg11-depleted larvae $(8 / 31)$ displayed $m m p 9$ expression similar to that shown for control MO-injected. (e-g) Quantification of Lyz ${ }^{+}$neutrophils within wounded control MO-injected and MMP9-depleted (e), DMSO- and GM6001-treated (f), and control MO-injected and Irg1l-depleted (g) Tg(lyz:EGFP) larvae at 0, 2, 4 and 6 hpw (mean \pm s.d.; $n=2$ separate wounded cohorts). Neutrophils quantified within wound region extending $100 \mu \mathrm{m}$ from wound border. (h,i) Individual neutrophil tracks within wounded control MO-injected and Irg1l-depleted $\operatorname{Tg}($ lyz:EGFP) larvae, respectively (lateral views, anterior to left). Circles highlight tracked neutrophils at the end of tracks. (j-I) Track velocities, displacement rates and meandering indices, respectively, of individual neutrophil tracks, as detected in $\mathbf{h}$ and $\mathbf{i}$ (mean \pm s.d.; $n=3$ individual control MO-injected and Irg1l-depleted wounded larvae). ( $\mathbf{m}$ ) Neutrophil tracks within wounded $T g$ (lyz:EGFP)/Tg(ef1- $\alpha$ :DsRED2) double transgenic larvae (from $15 \mathrm{~h}$ time-lapse experiment) with tracks pseudo-colored to reflect $z$ axis depth (colder colours represent tracks closer to viewer). Lateral view, anterior to left. (n) 3D transverse view of $\mathbf{m}$ revealing neutrophils migrating over surface of red-fluorescent somites (white arrowhead) en route to wound. White and black asterisks mark wound border. Scale bars, $20 \mu \mathrm{m}$ in $\mathbf{b} ; 50 \mu \mathrm{m}$ in $\mathbf{h}$ and $\mathbf{m}$. Statistical significance determined using a Student's $t$-test. CHT, caudal hematopoietic tissue; ${ }^{\star} P$-value $<0.05$; ${ }^{\star \star} P$-value $<0.01$; ${ }^{\star \star \star} P$-value $<0.001$; ${ }_{\star \star \star \star \star} P$-value $<0.0001$.
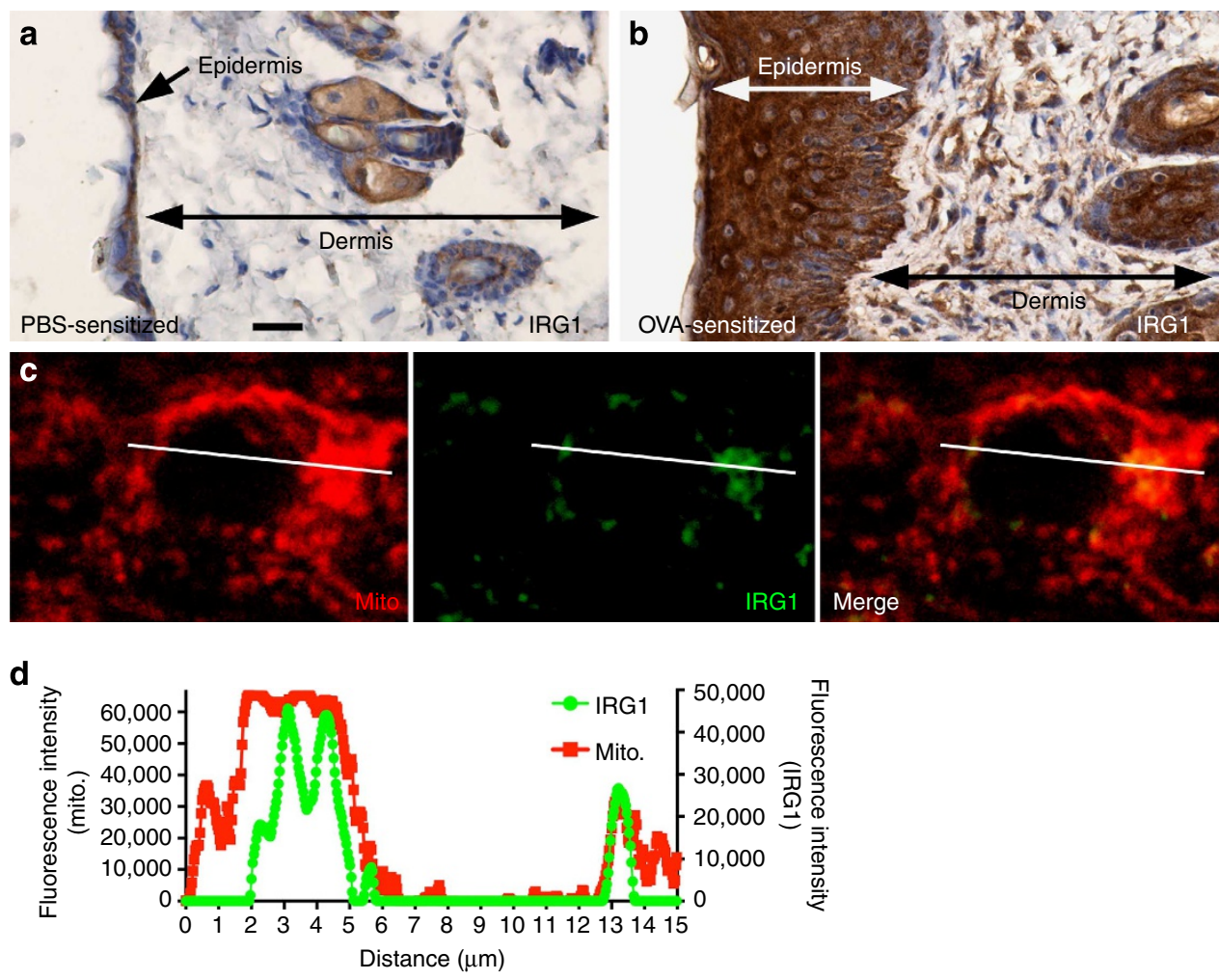

Figure 7 | Murine IRG1 is detected within epidermal cells in a mouse model of atopic dermatitis and localizes to the mitochondria.

(a,b) Immunohistochemical staining for IRG1 within skin biopsies taken from PBS-sensitized (a) and OVA-sensitized (b) mice, respectively.

(c) Immunofluorescent detection of mitochondria and IRG1 within epidermis of OVA-sensitized mouse. (d) Line fluorescence intensity measurements, for line shown in c, for Mitochondria- and IRG1-specific signals demonstrating IRG1 associates with mitochondria. Representative images from $n=4$ separate PBS- and OVA-sensitized mice. Scale bars, $50 \mu \mathrm{m}$ in $\mathbf{a}, 5 \mu \mathrm{m}$ in $\mathbf{c}$.

neutrophils has provided unique insights into the migratory kinetics of these innate immune cells ${ }^{23,24,41}$. By live imaging $\operatorname{Tg}(l y z: E G F P)$ larvae we assessed whether Irg1l-regulated $m m p 9$ expression contributed to the recruitment of lysozyme (Lyz) ${ }^{+}$ neutrophils to wounded tissue. To first evaluate whether MMP9 contributes to neutrophil recruitment in our model, we assessed neutrophil recruitment to tail wounds within MMP9-depleted larvae and larvae treated with the pan-MMP inhibitor GM6001 (ref. 42). In contrast to control larvae, MMP9-depleted and GM6001-treated larvae possessed fewer neutrophils at the wound region (Fig. 6e,f and Supplementary Fig. 9), despite maintaining typical (whole-larva) numbers of neutrophils (Supplementary Fig. 10). A similar reduction was also observed within Irg1depleted larvae (Fig. 6g), despite maintaining typical (wholelarvae) numbers of neutrophils (Supplementary Fig. 10). Tracking individual cell trajectories within wounded $\operatorname{Tg}($ lyz:EGFP) larvae revealed that neutrophils migrated with significantly reduced velocities, displacement rates and meandering indices within Irg1-depleted larvae, when compared with control larvae (Fig. 6h-1). Of interest, when examining migration paths of 
neutrophils towards wounded tissue, most neutrophils migrated ventral to somitic tissue from the caudal hematopoietic tissue (a larval hematopoietic site). Those neutrophils that migrated more medially were observed to migrate superficially in close proximity to the irgll/mmp9-expressing epidermis rather than through deeper somitic tissues (Fig. 6m,n).
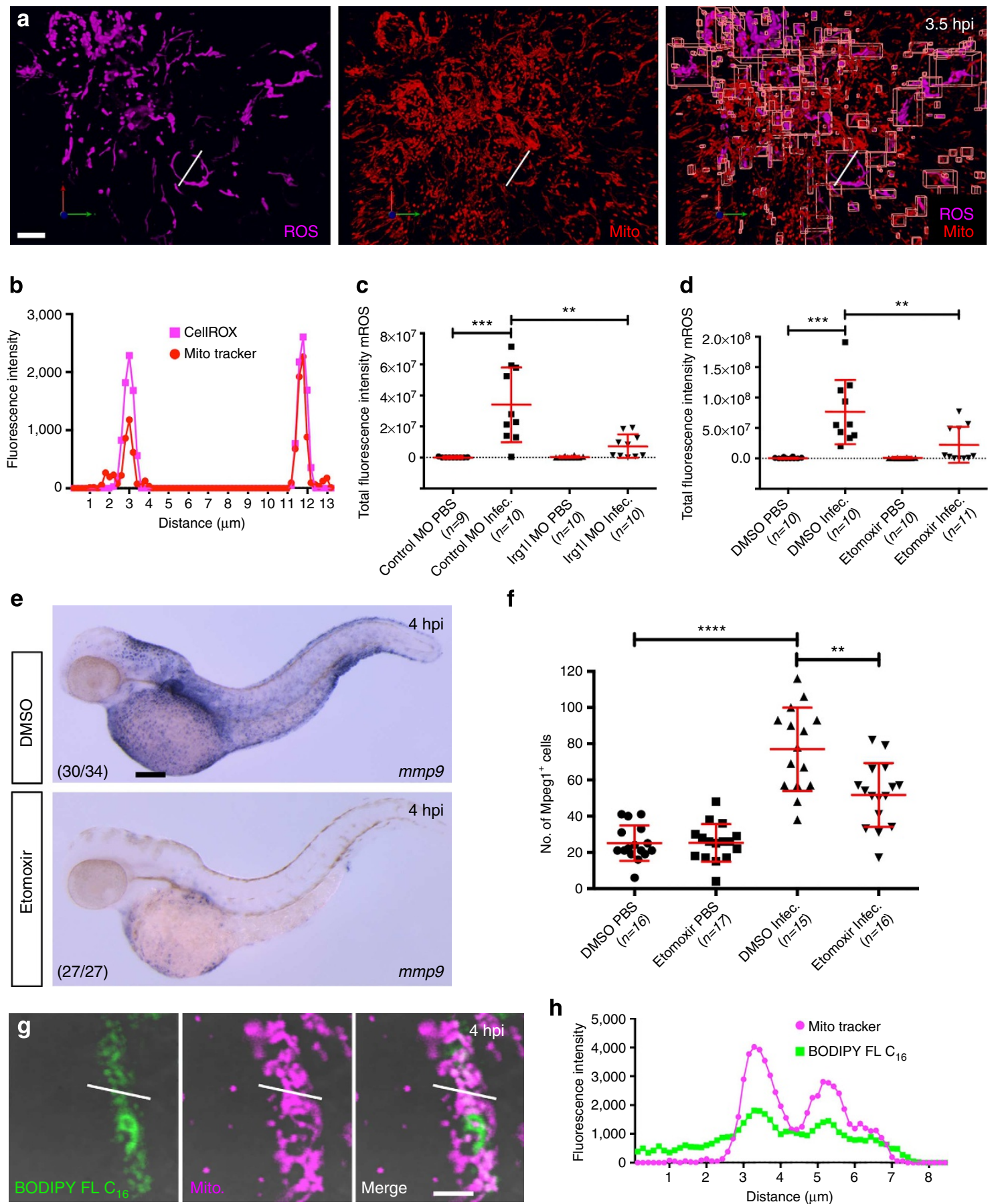

h
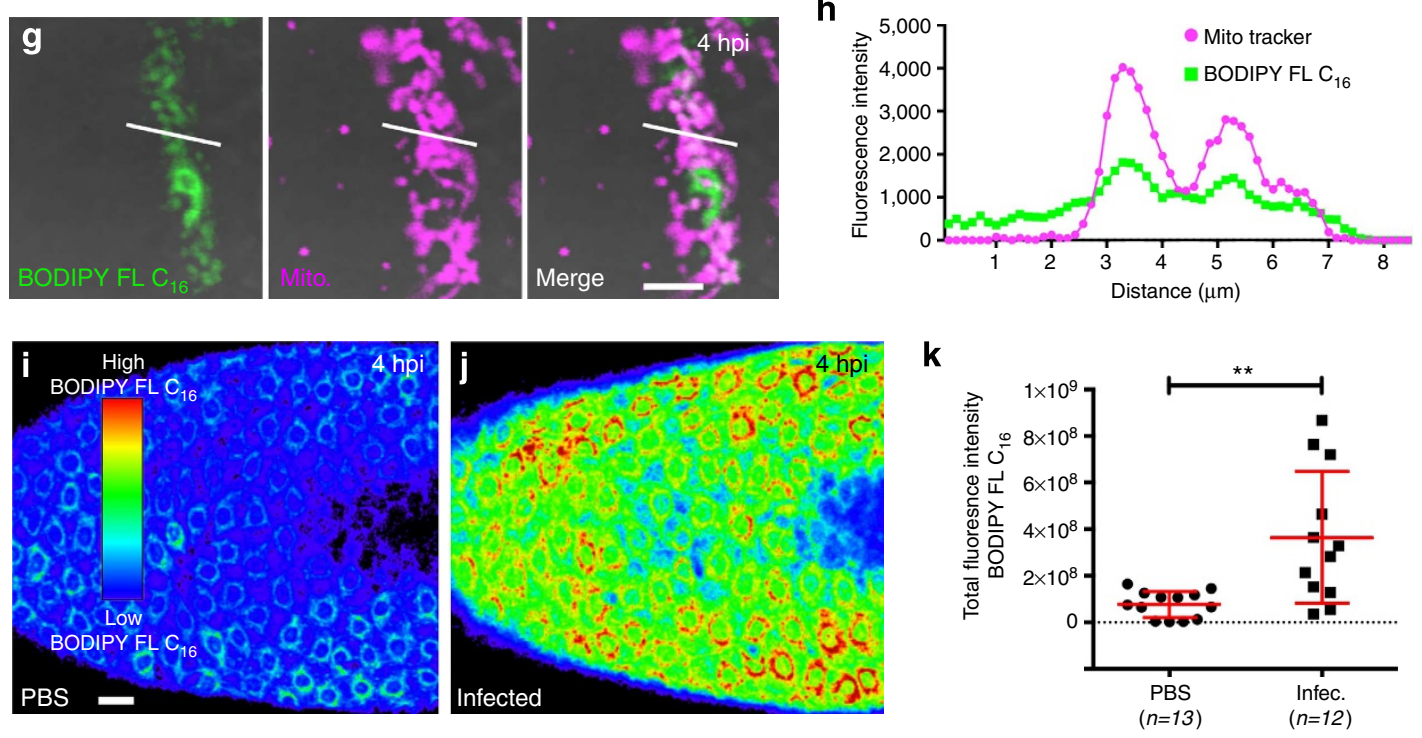
Together, these data suggest that, in addition to infectiondriven cutaneous inflammation, Irg1l also contributes to the timely recruitment of innate immune cells to wounded tissues through regulating $m m p 9$ expression within epidermal cells.

Mouse epidermal cells upregulate IRG1 during inflammation. Atopic dermatitis is an increasingly common allergic skin disease characterized by chronically relapsing cutaneous inflammation. Skin wash samples from atopic dermatitis lesions possess elevated levels of MMP9 and MMP activity, raising the possibility of a functional role for MMP9 during atopic dermatitis-associated inflammation $^{43}$. Elevated expression of IRG1 (the mammalian homologue of zebrafish Irg1l) has only been reported in the uterine epithelium during implantation, within immunestimulated monocytes/macrophages (where it localizes to the mitochondria) and 'neurotoxic' microglia ${ }^{29,44-46}$. We sought to explore whether IRG1 expression was also elevated within epidermal cells in a mouse model of atopic dermatitis.

Using an ovalbumin-mediated epicutaneous sensitization model of atopic dermatitis, we assessed IRG1 abundance within the inflamed skin by immunohistochemistry. Strong staining for IRG1 was detected in the thickened epidermis of ovalbuminsensitized mice (employing two different IRG1 antibodies), when compared with control (PBS-sensitized) skin biopsies (Fig. 7a,b and Supplementary Fig. 11a,b). Immunofluorescence revealed IRG1 was present within the mitochondria of epidermal cells (Fig. 7c,d).

These results confirm elevated expression of IRG1 within the inflamed epidermis in a mouse model of atopic dermatitis and support a conserved role for mammalian IRG1 during cutaneous inflammation.

Irg11 regulates $\beta$-oxidation-driven mROS production. Betaoxidation is the metabolic process where fatty acids are catabolized in the mitochondria to generate ATP through the OXPHOS pathway. As a by-product of OXPHOS, mitochondria produce ROS, in the form of superoxide, as electrons leak from the electron transport chain ${ }^{4}$. Studies have revealed that ROS can function as a signalling molecule to regulate the transcription and functional activity of various inflammatory mediators, including MMPs ${ }^{14,15,48}$. We have previously shown that the paralog of Irg1l, Irg1, is required within the mitochondria of activated macrophages for enhanced utilization of fatty acids that fuels mROS production ${ }^{7}$. We investigated whether Irg1l-dependent epidermal expression of $m m p 9$ could be explained by a functional role for Irg1l during $\beta$-oxidation-driven mROS production.
To first assess whether epidermal cells increased production of mROS following infection, we took advantage of the mitochondria-specific MitoTracker and ROS-sensitive CellROX fluorescent probes. Live imaging ROS within epidermal cells of larvae co-injected with Salmonella, MitoTracker and CellROX into the hindbrain ventricle showed that ROS was restricted to mitochondria (Fig. 8a,b). Quantifying overlapping CellROX/ MitoTracker fluorescence signal within epidermal cells overlying the midbrain/hindbrain infection site (measured as total fluorescence intensity of colocalized signal) revealed elevated mROS production, when compared with PBS-injected controls (Fig. 8c). Of note, similar analysis in the presence of the mitochondrial OXPHOS inhibitor rotenone abolished this infection-driven augmentation in ROS production, confirming the ROS detected was coupled to mitochondrial OXPHOS (Supplementary Fig. 12). Similar analysis within Irg1l-depleted larvae (Fig. 8c and Supplementary Fig. 13a) and larvae treated with an inhibitor of fatty acid $\beta$-oxidation, etomoxir (Fig. 8d and Supplementary Fig. 13b), demonstrated that this elevated mROS production was Irgll- and $\beta$-oxidation-dependent. Examining $m m p 9$ expression and macrophage recruitment within infected etomoxir-treated larvae revealed $\beta$-oxidation of fatty acids contributed to infection-responsive epidermal expression of $m m p 9$ and macrophage recruitment (Fig. 8e,f and Supplementary Fig. 14a,b). Of note, etomoxir-treated larvae maintained unaltered (whole-larva) numbers of macrophages (Supplementary Fig. 7).

These data suggest that, in response to infection, Irg1l helps regulate $\beta$-oxidation of fatty acids within the mitochondria of epidermal cells, resulting in elevated mROS-driven $m m p 9$ expression that contributes to macrophage recruitment.

Infection enhances mitochondrial uptake of fatty acids. We have previously live-imaged the subcellular trafficking of the fluorescent fatty acid analogue BODIPY FL $\mathrm{C}_{16}$ to show that mitochondria of activated macrophages enhance their uptake of fatty acids to fuel OXPHOS-coupled mROS production ${ }^{7}$. We have further demonstrated that this infection-responsive process requires the activity of the zebrafish paralog of Irg1l, Irg1. Our model for Irg1l function within epidermal cells predicts similar enhanced uptake of fatty acids by the mitochondria to fuel mROS production and subsequent MMP activity. To investigate whether epidermal cells enhance their uptake of fatty acids in response to infection, we investigated the subcellular distribution of BODIPY $\mathrm{FL} \mathrm{C}_{16}$ within epidermal cells overlying the midbrain/hindbrain infection site.

Immersion of larvae within BODIPY FL $\mathrm{C}_{16}$ and MitoTracker probe following injection of Salmonella into the hindbrain

Figure 8 | Irg1l regulates $\beta$-oxidation-driven mROS production within epidermal cells that elevate mitochondrial uptake of fatty acids following

infection. (a) Detection of mROS within epidermal cells overlying infection region. (b) Line fluorescence intensity measurements, for line shown in a, for CellROX and MitoTracker probes demonstrating overlapping peak fluorescence intensities. (c) Quantification of mROS within individual control MOinjected and Irg1l-depleted larvae following PBS control and Salmonella injection, as detected at 3.5 hpi (mean \pm s.d.; $n=2$ separate infection experiments). (d) Quantification of mROS (as in c) within individual DMSO- and etomoxir-treated larvae injected with PBS or Salmonella (mean \pm s.d.; $n=2$ separate infection experiments). (e) Expression of $\mathrm{mmp} 9$ within DMSO- and etomoxir-treated larvae following infection, as detected at 4 hpi $(n=2$ separate infection experiments, lateral views, anterior to left). Remainder of infected DMSO-treated larvae (4/34) displayed weak epidermal mmp9 expression. (f) Quantification of $\mathrm{Mpeg}^{+}$macrophages within the midbrain/hindbrain region of DMSO- and etomoxir-treated $\operatorname{Tg}(\mathrm{mpeg}$ : EGFP) larvae at 4 hpi, following PBS or Salmonella injections (mean \pm s.d.; $n=2$ separate infection experiments). (g) Live imaging of BODIPY FL $C_{16}$ and MitoTracker probe within epidermal cells overlying the midbrain/hindbrain region following Salmonella injection. (h) Line fluorescence intensity measurements, for line shown in $\mathbf{g}$, for BODIPY FL $\mathrm{C}_{16}$ and MitoTracker probes demonstrating overlapping peak fluorescence intensities. (i,j) Live imaging mitochondrial uptake of BODIPY FL $\mathrm{C}_{16}$ within epidermal cells overlying the midbrain/hindbrain region following PBS (i) or Salmonella (j) injection, as detected at $4 \mathrm{hpi}$ (dorsal views, anterior to left). BODIPY FL $\mathrm{C}_{16}$ fluorescence intensity is displayed as a colormap, with warmer colours representing higher signal intensities. (k) Quantification of total fluorescence intensities of BODIPY FL $\mathrm{C}_{16}$ within epidermal cells overlaying the midbrain/hindbrain region for individual larvae as shown in $\mathbf{i}$ and $\mathbf{j}$ (mean \pm s.d.; $n=2$ separate infection experiments). Numbers represent frequencies of larvae with displayed phenotypes. Scale bars, $10 \mu \mathrm{m}$ in $\mathbf{a} ; 100 \mu \mathrm{m}$ in e; $5 \mu \mathrm{m}$ in $\mathbf{g} ; 20 \mu \mathrm{m}$ in i. Statistical significance determined using a Student's $t$-test. ${ }^{\star \star} P$-value $<0.01$; ${ }^{\star \star \star} P$-value $<0.001 ;{ }^{\star \star \star \star} P$-value $<0.0001$. 

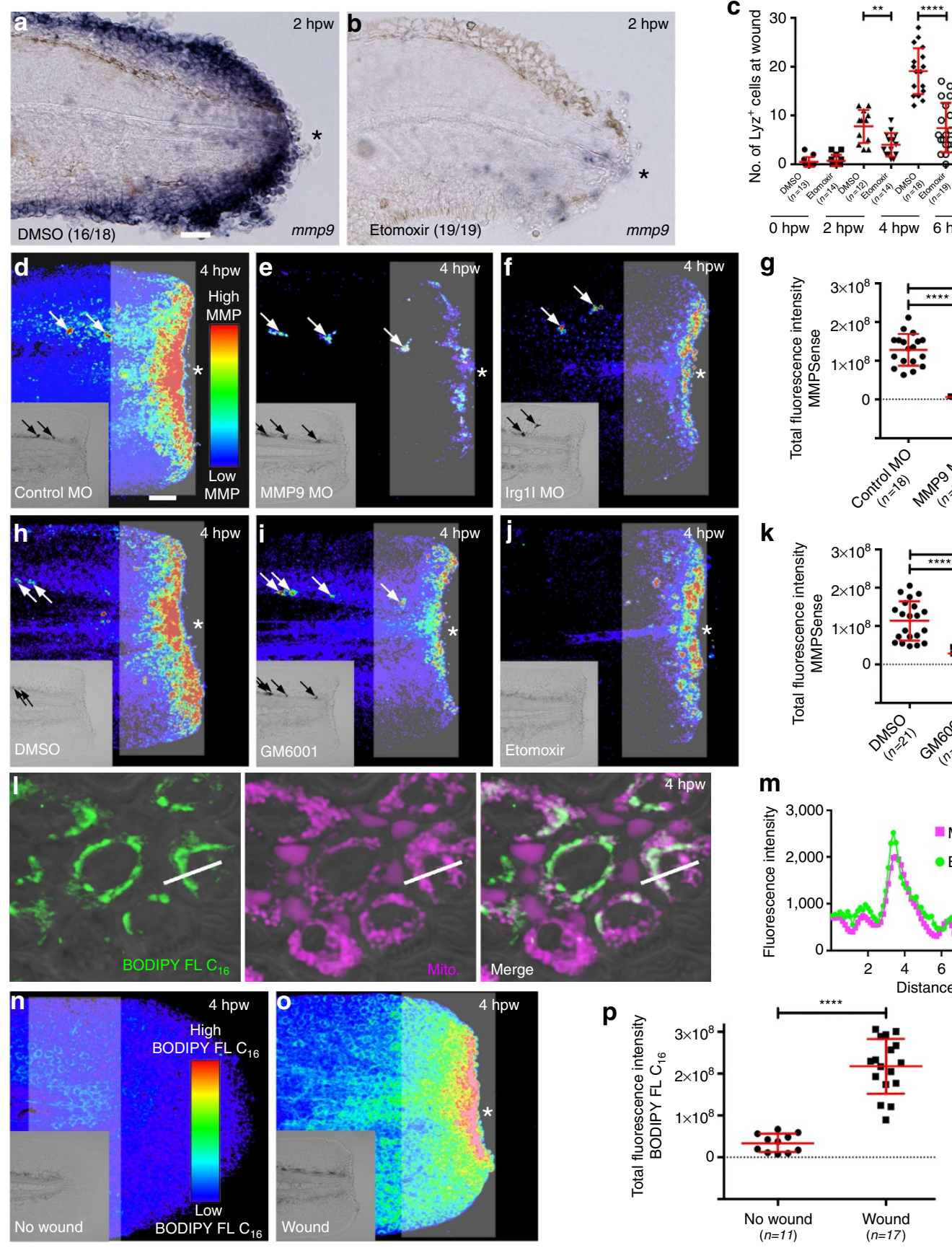

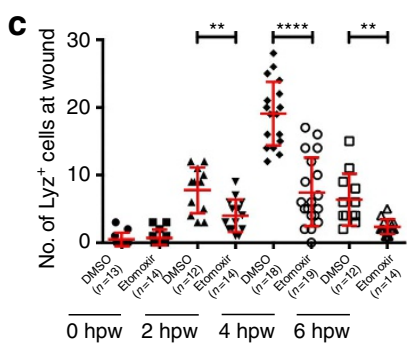

g

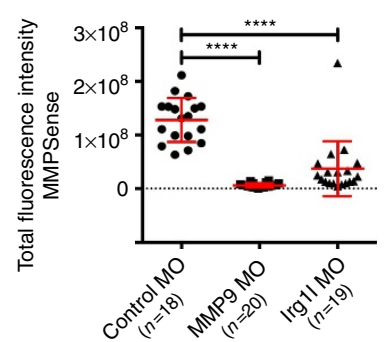

k

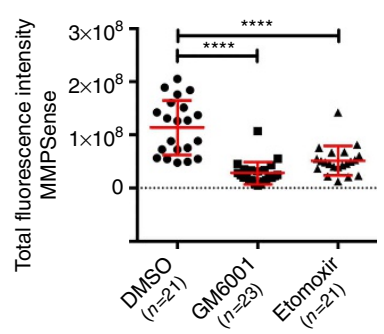

m

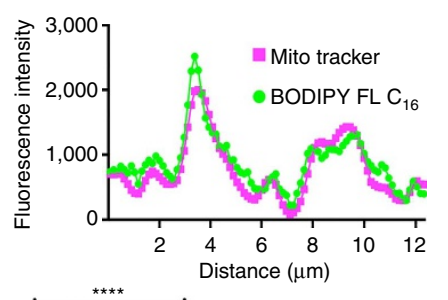

$(n=11)$

Figure 9 | $\beta$-oxidation and Irg1| contribute to MMP production by epidermal cells that elevate mitochondrial uptake of fatty acids following wounding.

$(\mathbf{a}, \mathbf{b})$ Expression of $\mathrm{mmp} 9$ within wounded DMSO- and etomoxir-treated larvae, respectively, at $2 \mathrm{hpw}$ ( $n=2$ separate wounded cohorts). Remainder of wounded DMSO-treated larvae (2/18) displayed weak mmp9 expression. (c) Quantification of Lyz ${ }^{+}$neutrophils within wounded DMSO- and etomoxir-treated $\operatorname{Tg}($ lyz:EGFP) larvae at 0, 2, 4 and 6 hpw (mean \pm s.d.; $n=2$ separate wounded cohorts). (d-f) Live imaging MMP activity (using MMPSense 645 FAST reagent) within control MO-injected, MMP9-depleted and Irg1l-depleted wounded larvae, as detected at 4 hpw. Fluorescence intensity is displayed as a colormap, with warmer colours representing higher MMP activity. Arrows highlight pigment cell auto-fluorescence. Insets show bright field views of wounds. (g) Quantification of total fluorescence intensities of MMPSense within individual larvae as shown in $\mathbf{d}-\mathbf{f}$ (mean \pm s.d.; $n=2$ separate wounded cohorts). White boxes in d-f highlight measured region. (h-k) Live imaging and quantification of MMP activity within DMSO-, GM6001- and etomoxir-treated wounded larvae, as detected and quantified in $\mathbf{d}-\mathbf{g}$ (mean \pm s.d.; $n=2$ separate wounded cohorts). (I) Live imaging BODIPY FL $\mathrm{C}_{16}$ and MitoTracker probe within epidermal cells at the wound border, as detected at $4 \mathrm{hpw}$. (m) Line fluorescence intensity measurements, for line shown in $\mathbf{I}$, for BODIPY FL $\mathrm{C}_{16}$ and MitoTracker probes demonstrating overlapping peak fluorescence intensities. (n,o) Live imaging mitochondrial uptake of BODIPY FL $\mathrm{C}_{16}$ within epidermal cells in non-wounded (n) or wounded (o) larvae, at $4 \mathrm{hpw}$. BODIPY FL $\mathrm{C}_{16}$ fluorescence intensity is displayed as a colormap. (p) Quantification of total fluorescence intensities of BODIPY FL $\mathrm{C}_{16}$ within epidermal cells of individual larvae as shown in $\mathbf{n}$ and $\mathbf{0}$ (mean $\pm \mathrm{s}$.d.; $n=2$ separate wounded cohorts). Measurements taken within regions highlighted with white box. White and black asterisks mark wound border. Numbers represent frequencies of larvae with displayed phenotypes. Scale bars, $20 \mu \mathrm{m}$ in $\mathbf{a} ; 50 \mu \mathrm{m}$ and d; $5 \mu \mathrm{m}$ in I. Statistical significance determined using a Student's t-test. ${ }^{\star \star} P$-value $<0.01 ;{ }^{\star \star \star \star} P$-value $<0.0001$. 
ventricle revealed mitochondrial uptake of the fluorescent fatty acid analogue within overlying epidermal cells, as detected at $4 \mathrm{hpi}$ (Fig. 8g,h). Of note, although BODIPY FL $\mathrm{C}_{16}$ largely colocalized to the mitochondria, in some instances fluorescent signal was detected outside of the mitochondria, albeit in close proximity (Fig. 8g). This likely reflects the dynamic process of fatty acid transport to the mitochondria. Further supporting the mitochondrial uptake of BODIPY $\mathrm{FL} \mathrm{C}_{16}$, mitochondrial localization within epidermal cells following infection was disrupted following treatment with etomoxir (Supplementary Fig. 15a,b). Etomoxir functions as an inhibitor of carnitine palmitoyltransferase-1 (ref. 49), a rate-limiting enzyme essential for the transport of fatty acids across the outer mitochondrial membrane. Quantifying the total fluorescence intensity of BODIPY FL $\mathrm{C}_{16}$ within these cells showed enhanced mitochondrial uptake following infection, when compared with PBS-injected controls (Fig. 8i-k).

These data suggest that, following infection, elevated uptake and $\beta$-oxidation of fatty acids within the mitochondria of epidermal cells fuels mROS production through an Irg1l-dependent mechanism. Enhanced mROS-driven mmp9 expression then contributes to macrophage recruitment.

及-oxidation contributes to MMP production following wounding. We next investigated whether similar enhanced fatty acid $\beta$-oxidation was driving Irg1l-dependent $m m p 9$ expression and neutrophil migration within our wound-induced model of cutaneous inflammation.

Similar to Irg1l-depleted larvae (Fig. 6c,d,g), etomoxir treatment resulted in a marked reduction in wound-induced epithelial mmp9 expression (Fig. 9a,b) and a significant reduction in neutrophil recruitment to tail fin wounds (Fig. 9c), despite maintaining unaltered whole-larvae numbers of $\mathrm{Lyz}^{+}$neutrophils (Supplementary Fig. 10). We next exploited the live imaging potential of the MMPSense FAST fluorescent probe that produces fluorescence following cleavage by $\mathrm{MMPs}^{50}$, to live image MMP activity within tail fin wounds. Measuring the total fluorescence signal of MMPSense within a defined wound region $(150 \mu \mathrm{m}$ from the wound border), at $4 \mathrm{hpw}$, we detected wound-specific MMP activity that was both MMP9- and Irg1l-dependent (Fig. 9d-g). This fluorescent signal at the wound region was also significantly diminished in the presence of the pan-MMP inhibitor GM6001 and etomoxir (Fig. 9h-k). Zebrafish leukocytes express both $m m p 9$ and $m m p 13$ (refs 36,51), suggesting that they are a source of MMP activity. To confirm that the primary source of wound-induced MMP we were detecting and measuring using the MMPSense probe was epidermal, we quantified the percentage of total fluorescence intensity that was neutrophiland macrophage-specific. Live imaging and quantification of MMPSense fluorescent signal within $\operatorname{Tg}($ lyz:EGFP) and $\mathrm{Tg}$ (mpeg1:EGFP) larvae revealed that only $0.012 \pm 0.027 \%$ $(n=11$ larvae $)$ and $2.05 \pm 2.26 \%(n=11$ larvae $)$ of the total fluorescence intensity within the wound region colocalized with $\mathrm{Lyz}^{+}$neutrophils and Mpeg1 ${ }^{+}$macrophages, respectively (Supplementary Fig. 16).

Wounding enhances mitochondrial uptake of fatty acids. To evaluate whether the mitochondria of epidermal cells enhanced their uptake of fatty acids following wounding, similar to that observed following infection (Fig. $8 \mathrm{~g}-\mathrm{k}$ ), we live-imaged the subcellular trafficking of BODIPY FL $\mathrm{C}_{16}$ within epidermal cells following wounding. When compared with an equivalent anatomical position within the tail of non-wounded larvae, epidermal cells of wounded larvae demonstrated enhanced mitochondrial uptake of BODIPY FL $\mathrm{C}_{16}$ (Fig. 91-p) within the wound region (150 $\mu \mathrm{m}$ from the wound border). Of note, mitochondrial uptake of BODIPY FL $\mathrm{C}_{16}$ was again inhibited following etomoxir treatment (Supplementary Fig. 15c,d).

Similar to our infection-induced inflammation model, these results suggest that elevated uptake and $\beta$-oxidation of fatty acids within the mitochondria of epidermal cells following wounding fuels MMP production through an Irg1l-dependent mechanism. Enhanced MMP activity then contributes to the timely recruitment of neutrophils to the wound region.

\section{Discussion}

By exploiting the live-imaging potential of embryonic zebrafish to dissect the function of Irg11, we have uncovered a new mechanism by which epidermal cells help direct the recruitment of leukocytes during inflammation. Using an infection-induced model of cutaneous inflammation we show that infectionresponsive expression of $\mathrm{irg} 1 \mathrm{l}$ within epidermal cells is MyD88dependent and GC-sensitive. We predict that resident MyD88 ${ }^{+}$ macrophages stimulate epidermal irg1l expression via secreted cytokines. Although strongest in epidermal cells overlying the infection site, epidermal expression of irgll (and $\mathrm{mmp9}$ ) was detected throughout the entire larvae following infection, suggesting that this macrophage-derived signal acts systemically. A previous study has shown zebrafish Cxcl8 (IL8), secreted from an unidentified cellular source at the infection site, acts systemically to trigger the rapid recruitment of leukocytes from the caudal hematopoietic tissue $\mathrm{e}^{52}$. Investigating whether a similar systemically acting chemokine/chemokine receptor partnership operates in our model to drive epidermal expression of $\mathrm{irgll}$ is a focus of future research. Of interest, infection-induced upregulation of irg1l expression within $\mathrm{GR} / \mathrm{Nr} 3 \mathrm{cl}{ }^{+}$ oropharyngeal tissues was not suppressed following GC treatment, suggesting that the sensitivity of irgll expression to GC-mediated suppression is tissue-specific. We provide evidence that Irg1l is necessary for the clearance of injected bacteria through helping regulate enhanced fatty acid $\beta$-oxidation to fuel mROS-driven mmp9 expression within epidermal cells, which helps direct further macrophage recruitment (Fig. 10). We further reveal that a similar Irg1l-dependent immunometabolic mechanism operates within epidermal cells during woundinduced inflammation to help orchestrate the timely recruitment of neutrophils (Fig. 10). In addition to uncovering a new immunometabolic function for epidermal cells during inflammation, this study represents the first whole-animal liveimaging investigation of epidermal cell metabolism during inflammation.

Zebrafish possess co-orthologues for IRG1 (irg1 and irg1l). Our previous study has established a role for zebrafish Irg1 in coupling metabolism with immune cell function within macrophages ${ }^{f}$. We showed that irg1 was specifically expressed within immunestimulated macrophages where the encoded enzyme was required for enhanced $\beta$-oxidation of fatty acids to fuel OXPHOS-coupled mROS production. Here we demonstrate a similar role for Irg1l during enhanced utilization of fatty acids to fuel mROS production within epidermal cells during infection- and woundinduced inflammation. To date, mammalian IRG1 expression has been reported in activated macrophages, neurotoxic microglia and within the uterine epithelium ${ }^{44,46,53}$. IRG1 has been shown to localize to the mitochondria using fluorescent fusion proteins and cell fractionation experiments ${ }^{45}$. Functional experiments further support a mitochondrial association ${ }^{7,54}$. Employing a mouse model of atopic dermatitis and antibodies against human IRG1, we have shown elevated levels of IRG1, as detected by immunofluorescence, within the inflamed skin of ovalbumin- 


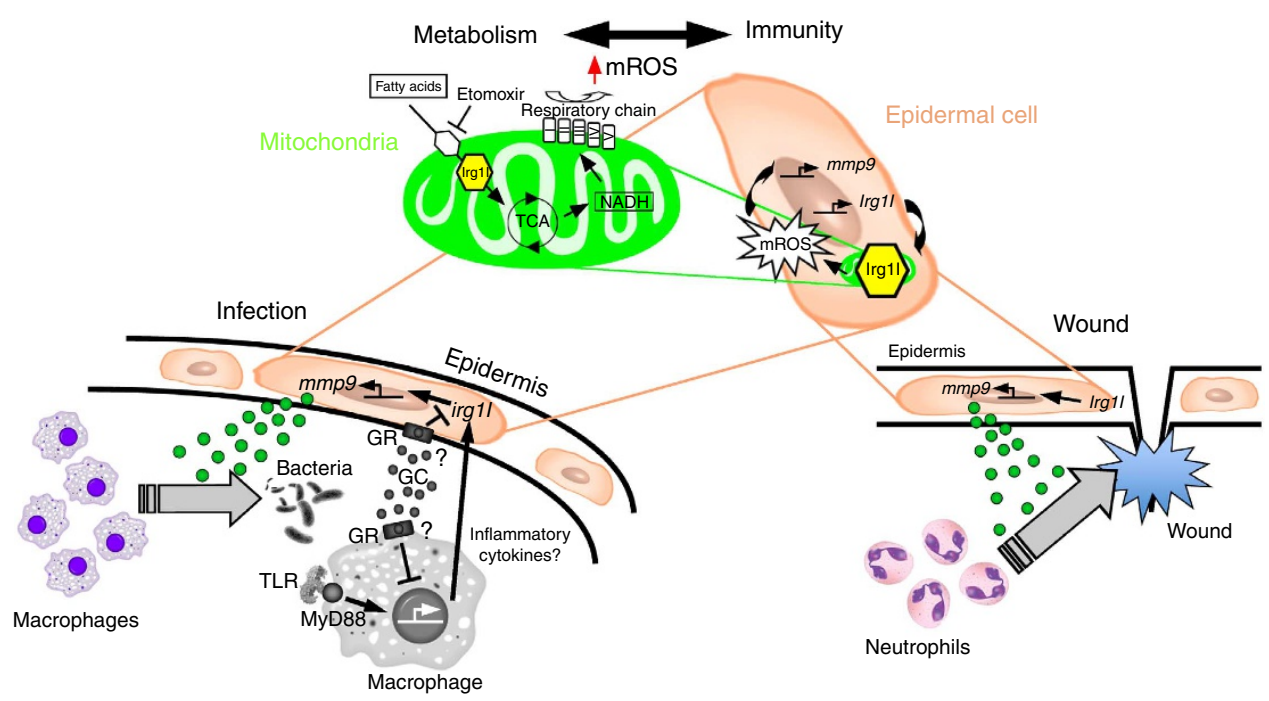

Figure 10 | Proposed model describing function of Irg1l within epidermal cells following infection and wounding. In response to infection, resident

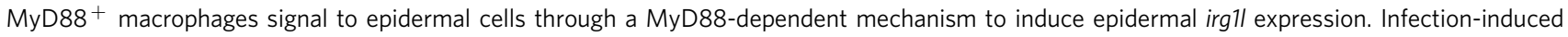
epidermal expression of $\mathrm{irg} 1 \mathrm{l}$ is suppressed by the anti-inflammatory activity of GCs on $\mathrm{GR}^{+}\left(\mathrm{Nr}_{\mathrm{C}} 1^{+}\right)$macrophages and/or epidermal cells. Irg1l helps couple metabolism and immunity within epidermal cells through regulating fatty acid-fuelled production of mROS that contributes to epidermal mmp 9 expression and MMP-mediated recruitment of macrophages. In response to wounding, epidermal cells similarly couple Irg1l-dependent fatty acid-fuelled production of mROS with mmp9 expression and MMP-mediated recruitment of neutrophils. Signalling components specific to infection-induced irg1l expression are shown in grey. TLR, toll-like receptor; GR, glucocorticoid receptor; GC, glucocorticoid; TCA, tricarboxylic acid cycle (Krebs cycle).

sensitized mice, where it associates with the mitochondria of keratinocytes. Our model predicts IRG1 is required for enhanced $\beta$-oxidation of fatty acids within keratinocytes during inflammation. In all eukaryotic organisms, $\beta$-oxidation occurs in mitochondria and peroxisomes ${ }^{55}$. It is emerging that, in response to inflammatory stress, mitochondria are highly dynamic and can form intimate physical interactions with other organelles (including peroxisomes and mitochondrial-associated endoplasmic reticulum membranes $)^{56}$. These interactions are believed to form signalling scaffolds that help coordinate immune cell function. Identifying how IRG1 associates with the mitochondria in our mouse dermatitis model, by generating mouse-specific IRG1 antibodies and performing immunogold labelling/transmission electron microscopy, is a focus of future research. Our results support a conserved immunometabolic role for IRG1 within mammalian epidermal cells during cutaneous inflammation and suggest that the function of mammalian IRG1 is subfunctionally partitioned between Irg1 and Irg1l in zebrafish. Subfunctional partitioning is common with duplicated genes resulting from the pre-teleost genome duplication. Occupying separate loci, duplicate genes are independently mutable and can acquire mutations within regulatory elements that help direct tissue-specific expression. This can lead to paralogs with tissuespecific functions that cumulatively recapitulate that of the ancestral gene ${ }^{57}$. Unlike ray-finned fish and reptiles, mammals possess one IRG1 orthologue (http://www.ensembl.org/ Danio_rerio/Gene/Compara_Tree?g=ENSDARG00000069844; $\mathrm{r}=9: 22866973-22871390$ ), suggesting that degenerative mutations in coding and/or regulatory regions (nonfunctionalization) have contributed to the loss of one of the gene duplicates in this lineage, a fate that accounts for most duplicated genes ${ }^{58}$.

Our model predicts that, during cutaneous inflammation, elevated $\beta$-oxidation of fatty acids within epidermal cells contributes to the recruitment of leukocytes through mROSdriven augmentation of $m m p 9$ expression. MMPs are wellrecognized mediators of inflammation and innate immune cell activity $^{59}$. By directly modulating chemokine gradients and degrading ECM components, MMPs shape the outcome of inflammation by helping direct the recruitment of innate immune effector cells (including neutrophils and monocytes/ macrophages) to sites of infection or injury ${ }^{59}$. During cutaneous inflammation, keratinocytes are a major source of MMP activity (including that provided by MMP-9) that, in addition to regulating re-epithelialization, are believed to contribute to leukocyte recruitment ${ }^{13,59}$. Supporting a connection between mitochondrial redox signalling and MMP activity, evidence suggests that mROS-sensitive signalling pathways direct both the expression and activity of MMP family members ${ }^{14}$. Furthermore, heat shock-induced MMP-9 expression within a human keratinocyte cell line is, in part, regulated by $\operatorname{mROS}^{60}$, and TNF- $\alpha$-stimulated human keratinocytes enhance production of MMP-9 through a ROS/NF- $\mathrm{BB}$-dependent mechanism ${ }^{15}$. Our study reveals the contribution of mROS-driven MMP activity towards leukocyte recruitment is fuelled by metabolic reprogramming in keratinocytes towards elevated fatty acid $\beta$-oxidation.

Supporting a connection between elevated $\beta$-oxidation and the inflammatory response during cutaneous inflammation, the expression of enzymes associated with fatty acid oxidation are elevated within the skin of patients with the inflammatory dermatosis psoriasis ${ }^{61,62}$. It has also been suggested that epidermal fatty acid-binding protein contributes to the pathogenesis of psoriasis through activation of the NF- $\kappa$ B signalling pathway ${ }^{63}$, a powerful transcriptional regulator of pro-inflammatory genes. Furthermore, etomoxir-mediated $\beta$-oxidation inhibition is beneficial in the treatment of psoriasis ${ }^{64}$. We provide a mechanistic link connecting enhanced fatty acid $\beta$-oxidation within epidermal cells to their immune function that acts to direct leukocyte recruitment. The precise mechanism through which Irg1l/IRG1 participates during $\beta$-oxidation-fuelled augmentation of mROS is the focus of future work.

Metabolic reprogramming as a strategy to regulate the immune response is known to operate within immune cells ${ }^{3,4}$. To our knowledge, this study describes the first example of metabolic reprogramming being employed within a non-immune cell type 
to help shape its contribution to the immune response. Pharmacologic targeting of this metabolic-immunological interface within keratinocytes may prove efficacious in treating inflammatory dermatoses.

\section{Methods}

Zebrafish maintenance. Zebrafish (Danio rerio) embryos were obtained from natural spawnings and raised at $28{ }^{\circ} \mathrm{C}$ in E3 Medium supplemented with $0.003 \%$ phenylthiourea (PTU) to inhibit pigmentation. The Tg(mpeg1:Gal4-VP16) $)^{g / 24}$; Tg(UAS-E1b:Kaede $)^{s 1999 t}$ (herein referred to as Tg(mpeg1:Gal4/UAS:Kaede)), $\operatorname{Tg}(m p e g 1: E G F P)^{g / 22}, \operatorname{Tg}(\text { mpeg1:mCherry })^{g l 23}, \operatorname{Tg}(\text { lyz:EGFP })^{N Z 117}$ and $\operatorname{Tg}(e f 1-\alpha$ : DsRED2) transgenic reporter lines were used in this study. Research was conducted with approval from the University of Auckland Animal Ethics Committee.

Mice. Six to 8-week-old female C57BL/6 mice were bred and housed in the Animal Resource Unit, Faculty of Medical and Health Sciences, University of Auckland, Auckland, New Zealand. The mice were kept in an air-conditioned room with controlled humidity, temperature and a $12 \mathrm{~h}$ light/dark cycle. All experiments were conducted under a protocol approved by the University of Auckland Animal Ethics Committee.

\section{Live bacterial and LPS injection of zebrafish larvae. Zebafish larvae were injected with non-fluorescent wild-type or GFP-expressing S. enterica serovar Typhimurium into the hindbrain ventricle as previously described ${ }^{31}$. In brief, anaesthetized $2 \mathrm{dpf}$ larvae were mounted in 3\% methyl cellulose (in E3 media) and injected with $1 \mathrm{nl}$ of bacterial injection preparation $(\sim 600 \mathrm{cfu} / \mathrm{nl}$ in filter-sterilized PBS) into the hindbrain ventricle. Immediately before and after injecting a cohort of larvae, an injection bolus was injected into filter-sterilized PBS (in duplicate) that was plated at 1:10 and 1:100 dilutions to quantify and validate infection dose. LPS (E. coli $\mathrm{K} 12$, InvivoGen) was similarly injected $\left(0.8 \mathrm{mg} \mathrm{ml}^{-1}, 1 \mathrm{nl}\right)$, as previously described $^{7}$.}

Microarray and analysis. Total RNA was extracted from dissected trunks of infected and PBS control-injected larvae at 2 dpi (injected at $2 \mathrm{dpf}$ ) using TRIzol reagent (Invitrogen) and purified using Qiagen RNeasy columns as per the manufacturer's instructions. Hybridization to Affymetrix GeneChip Zebrafish Genome arrays and data acquisition were performed at the Centre for Genomics and Proteomics, School of Biological Sciences, University of Auckland. Data were normalized using the RMA method and statistical analysis of differential expression between control and infected larval zebrafish trunks was performed using linear models (LIMMA).

Whole-mount in situ hybridization. WMISH was performed using digoxigenin and fluorescein-labelled riboprobes (Roche) and BM purple (Roche) colour precipitation or Tyramide Signal Amplification (Invitrogen) for fluorescent WMISH analysis, as previously described ${ }^{31,65}$

RT-PCR. Total RNA was extracted from embryos and larvae using TRIzol reagent (Invitrogen) as per the manufacturer's instructions. Superscript III reverse transcriptase (Invitrogen)-generated cDNAs were used to produce gene-specific amplicons using primer pairs designed to span at least 1 intron to control for contaminating genomic DNA. To assess Irg1l and MMP9 MO specificities the following (forward/reverse) primer pairs were used, $5^{\prime}$-TCCAGATCCTGACCAC AAC- $3^{\prime} / 5^{\prime}$-TTATTGCAGTTGGGCAAGC- $3^{\prime}$ and $5^{\prime}$-CTGATCTGATCTGAGC ACTTGC- $3^{\prime} / 5^{\prime}$-GCTGACGGACAAATATGCCTAAC- $3^{\prime}$ respectively. Primers to ef $1-\alpha\left(5^{\prime}\right.$-AAGGAGAAGATCGACCGTCG- $3^{\prime}$ and $5^{\prime}$-TGATTGAGGGAAATTC ACTTGG-3') were used as an internal control. To generate irgll-, krt4- and $m m p$ 9-targeting riboprobes the following primer pairs were used: $\mathrm{irg} 1 \mathrm{l}, 5^{\prime}$-TCC AGATCCTGACCACAAC- $3^{\prime}$ and $5^{\prime}$-TTATTGCAGTTGGGCAAGC- $3^{\prime}$; $k r t 4$ $5^{\prime}$-ATGGGCTTTGTTGCGGCTCCTATC- ${ }^{\prime}$ and $5^{\prime}$-TGAGTCGTAAGAAGGTT CAGAAGGG-3'; $m m p 9,5^{\prime}$-CGACTCAAGATGAGACTTGGAG- $3^{\prime}$ and $5^{\prime}$-GCT GACGGACAAATATGCCTAAC- $3^{\prime}$. Riboprobes to detect $m y d 88$ were as previously described ${ }^{18}$.

MO injection. MOs (Gene Tools, Philomath, OR, USA) were resuspended in sterile water and injected in $1 \times$ Danieau's solution. Efficacious doses for all MOs were determined empirically. RT-PCR was used to determine MO specificity where splice-blocking MOs were used. Sequences of MOs (and doses) are as follows: control MO, $5^{\prime}$-CCTCTTACCTCAGTTACAATTTATA- $3^{\prime}$ (1.5 pmole); $\mathrm{Nr} 3 \mathrm{c} 1$ MO (splice-blocking), $5^{\prime}$-GCCAGAGATATATGGAATACCTTCA- $3^{\prime}$ $\left(0.5\right.$ pmole $^{7}$; Irgll MO (splice-blocking), 5'-AACACATTAGGCAGTACTTACT GCT-3 $3^{\prime}$ (1.5 pmole); Nr5ala MO (translation-blocking), 5'-AATCCTCATCTG CTCTGAAGTC-3' $3^{\prime}\left(0.5\right.$ pmole) ${ }^{37}$; Irf8 MO (splice-blocking), 5'-AATGTTTCGC TTACTTTGAAAATGG-3 $3^{\prime}\left(1.5\right.$ pmole) ${ }^{33}$; MyD88 MO (splice-blocking), $5^{\prime}$-GGT TAAACACTGACCCTGTGGATC- $3^{\prime}$ (1.0 pmole) ${ }^{7}$; Gcsrf MO (splice-blocking), $5^{\prime}$-ATTCAAGCACATACTCACTTCCATT- $3^{\prime}(0.5 \text { pmole })^{31}$; Spil MO (translation-blocking), 5'-GATATACTGATACTCCATTGGTGGT-3' $(1.0 \text { pmole })^{66}$; MMP9 MO (splice-blocking), 5' -ATACAAAATACTCGACTCA CCTGCT-3' (0.25 pmole).

Confocal imaging. Live embryos and larvae were mounted for confocal imaging as previously described ${ }^{67}$. All live confocal imaging was performed on an Olympus FV1000 FluoView laser scanning confocal microscope equipped with an incubation chamber. Confocal imaging of fluorescent WMISH was performed using a Nikon C1 Eclipse confocal microscope. Images collected by confocal microscopy were processed and analysed using Volocity 6.1.1 (Perkin Elmer). To detect ROS production and mitochondria, the CellROX Deep Red Reagent (Invitrogen C10422, $1 \mathrm{nl}$ at $50 \mu \mathrm{M}$, absorption/emission maxima of $\sim 644 / 665 \mathrm{~nm}$ ) and MitoTracker Red CMXRos (Invitrogen M7512, $1 \mathrm{nl}$ at $20 \mu \mathrm{M}$, absorption/emission maxima of $\sim 579 / 599 \mathrm{~nm}$ ) fluorescent dyes were injected into the hindbrain ventricle, respectively. To live image BODIPY $\mathrm{FL}_{16}$ and mitochondria larvae were immersed in $50 \mu \mathrm{M}$ BODIPY FL $\mathrm{C}_{16}$ (Invitrogen, D-3821, imaged using absorption/emission maxima as used to detect Alexa Fluor $488(\sim 495 / 519 \mathrm{~nm})$ ) and $1 \mu \mathrm{M}$ MitoTracker Deep Red FM (Invitrogen M22426, absorption/emission maxima of $\sim 644 / 665 \mathrm{~nm}$ ), respectively, for $2 \mathrm{~h}$ immediately following infection or tail transection, washed for $30 \mathrm{~min}$ in PTU-treated E3 medium before mounting and imaging. To live image MMP activity, larvae were immersed in $40 \mu \mathrm{M}$ MMPSense 645 FAST (Perkin Elmer, NEV10100, absorption/emission maxima of $\sim 649 / 666 \mathrm{~nm}$ ) for $2 \mathrm{~h}$ immediately following tail transection, washed for $30 \mathrm{~min}$ in PTU-treated E3 medium before mounting and imaging. When quantifying mROS MMP activity and BODIPY FL $\mathrm{C}_{16}$ uptake using fluorescence intensity measurements, conservative laser excitation settings were used to ensure that we did not create/measure a saturated fluorescence signal. See Supplementary Methods for detailed descriptions of confocal imaging/analysis when used for neutrophil tracking and quantification of macrophage numbers at infection site, mROS production, BODIPY FL $\mathrm{C}_{16}$ fluorescence and MMPSense FAST fluorescence.

Chemical treatments. The following chemical agonists and antagonists were used. No abnormal development or toxicity was observed for all compounds used at the indicated working concentrations and routes of delivery. The GR antagonist RU-486 (Sigma, M8046) was delivered by immersion ( $2 \mathrm{~h}$ pre-treatment before infection, $250 \mu \mathrm{M} 1 \%$ DMSO). The $\beta$-oxidation inhibitor etomoxir (Sigma, E1905) was delivered by immersion (1-2h pre-treatment before infection or wounding, $100 \mu \mathrm{M}$ for infection and $10 \mu \mathrm{M}$ for wounding experiments, $1 \%$ DMSO). The OXPHOS inhibitor rotenone (Sigma, R8875) was delivered by immersion (1$2 \mathrm{~h}$ pre-treatment before infection, $1 \mu \mathrm{M}$ 1\% DMSO). The MMP inhibitor GM6001 (Sigma, M5939) was delivered by immersion (1-2 h pre-treatment before wounding, $200 \mu \mathrm{M} 1 \%$ DMSO). The GR agonists dexamethasone (Sigma), prednisolone (Sigma) and hydrocortisone (Sigma) were injected into the hindbrain ventricle $\left(1 \mathrm{nl}\right.$ at $\left.0.67 \mathrm{mg} \mathrm{ml}^{-1}\right)$ with PBS or Salmonella. Anti-inflammatory activities of dexamethasone, prednisolone and hydrocortisone (delivered by immersion at $\sim 5 \mu \mathrm{M}, 1 \% \mathrm{DMSO}$ ) were assessed by their ability to inhibit neutrophil migration using an acute tail-wounding assay with $3 \mathrm{dpf} T g(l y z$ :EGFP) larvae.

Quantitative PCR. qPCR was performed with Platinum SYBR Green qPCR SuperMix-UDG with ROX (Invitrogen) using an ABI PRISM 7900HT Fast sequence detection system (Applied Biosystems) as previously described ${ }^{68}$. Gene-specific oligonucleotides were designed using Primer Express software to detect irg $1 l\left(5^{\prime}\right.$-CACTAGATGTGGCAGAGCGT- $3^{\prime}$ and $5^{\prime}$-CCCAACTCCAATG CTGTCTA- $\left.3^{\prime}\right), m m p 9$ ( $5^{\prime}$-TGGGCACCTGCTCGTTGA- $3^{\prime}$ and $5^{\prime}$-TTGGAGAT GACCGCCTGC- $\left.3^{\prime}\right)$ and $e f 1-\alpha\left(5^{\prime}\right.$-TGCCTTCGTCCCAATTTCAG- $3^{\prime}$ and $5^{\prime}$-TAC CCTCCTTGCGCTCAATC- $\left.3^{\prime}\right)$. All reactions were performed in biological triplicate and technical quadruplicate and a melting curve analysis carried out to confirm reaction specificity. Analysis used the comparative CT method with ef $1-\alpha$ as an internal control.

Individual CFU counts. Individual CFU counts of larvae infected with Sal-GFP was performed as previously described ${ }^{69}$. Larval homogenates were plated in a dilution series on LB agar plates supplemented with $25 \mu \mathrm{g} \mathrm{ml}^{-1}$ kanamycin.

Acute tail wounding. An acute tail fin wound was generated by transecting the tail fin of anaesthetized $2 \mathrm{dpf}$ larvae using a sterile scalpel. Care was taken to transect tails at exactly the same anatomic location (posterior-most edge of notochord).

Flow cytometry. Flow cytometry was performed as previously described ${ }^{18}$ In brief, larvae were rinsed in calcium-free Ringer's solution for $15 \mathrm{~min}$ before removal of yolks by repeated passage through a $200 \mu$ l pipette tip. Larvae were then digested in $0.25 \%$ trypsin-EDTA (Gibco) for $2 \mathrm{~h}$ at $28.5^{\circ} \mathrm{C}$ with manual dissociation every $10 \mathrm{~min}$. The digestion was inhibited by the addition of $\mathrm{CaCl}_{2}$ to a concentration of $1 \mathrm{mM}$ and 5\% FBS. The dissociated cells were centrifuged for $5 \mathrm{~min}$ at 3,000 r.p.m. before resuspension in ice-cold $0.9 \times$ PBS, $5 \%$ FBS. Cells were 
passed several times through $40-\mu \mathrm{m}$ cell strainers (BD Falcon) before sorting. Flow cytometry was performed using a Becton Dickinson LSRII flow cytometer.

Immunofluorescence and immunohistochemistry. Immunohistochemical staining of paraffin sections was performed using the Vectastain elite ABC kit (Vector Laboratories, PK-6100) as per the manufacturer's instructions. Immunofluorescence was carried out as previously described ${ }^{18}$. Primary and secondary antibody combinations used were the following: chicken anti-GFP (1:500/Abcam, ab13970) with goat anti-chicken Alexa Fluor 488 (1:500/Invitrogen); rabbit anti-Kaede (1:500/MBL, PM012) with goat anti-rabbit Alexa Fluor 633 or goat anti-rabbit Alexa Fluor 488 (1:500/Invitrogen); rabbit anti-Human GR (P-20) (1:75/Santa Cruz Biotechnology Inc., sc-1002) with goat anti-rabbit Alexa Fluor 546 (1:500/Invitrogen); rabbit anti-human IRG1 (1:300/N-20, sc-84191 and C-18, sc-84189, Santa Cruz Biotech.) with goat anti-rabbit Alexa Fluor 488 (1:500/ Invitrogen) or biotinylated horse anti-rabbit IgG; mouse anti-mitochondria antibody (1:500/Abcam, ab3298) with goat anti-mouse Alexa Fluor 568 (1:500/ Invitrogen)

Ovalbumin-mediated epicutaneous sensitization model of AD. The protocol for inducing atopic dermatitis was as previously described ${ }^{70}$. In brief, mice were shaved, and the dorsal skin tape-stripped six times under anaesthesia. Ovalbumin $(100 \mu \mathrm{g}$ in $100 \mu \mathrm{l}$ of PBS) or PBS (as a negative control) was placed on a patch of gauze $(1 \times 1 \mathrm{~cm})$, which was secured to the skin site with a transparent bioocclusive dressing for a 1-week period and then removed. Each mouse received a total of three 1-week exposures to a patch separated by 2-week patch-free intervals. Mice were monitored daily to ensure that patches remained in place throughout the sensitization period. Mice were killed $24 \mathrm{~h}$ after removal of the third patch and skin tissue samples were collected. Skin tissues were fixed in $10 \%$ formalin, embedded into paraffin and sectioned.

Statistical analysis. Statistical analyses were performed using Prism 5.0 (GraphPad Software, Inc.). Statistical significance was assessed using unpaired, two-tailed $t$-tests and all data presented in scatter plots show means with s.d. $P$ values $<0.05$ were considered statistically significant.

\section{References}

1. Odegaard, J. I. \& Chawla, A. The immune system as a sensor of the metabolic state. Immunity 38, 644-654 (2013).

2. Mathis, D. \& Shoelson, S. E. Immunometabolism: an emerging frontier. Nat. Rev. Immunol. 11, 81-83 (2011).

3. Pearce, E. L. \& Pearce, E. J. Metabolic pathways in immune cell activation and quiescence. Immunity 38, 633-643 (2013).

4. Biswas, S. K. \& Mantovani, A. Orchestration of metabolism by macrophages. Cell Metab. 15, 432-437 (2012).

5. Chawla, A., Nguyen, K. D. \& Goh, Y. P. Macrophage-mediated inflammation in metabolic disease. Nat. Rev. Immunol. 11, 738-749 (2011).

6. West, A. P., Shadel, G. S. \& Ghosh, S. Mitochondria in innate immune responses. Nat. Rev. Immunol. 11, 389-402 (2011).

7. Hall, C. J. et al. Immunoresponsive gene 1 augments bactericidal activity of macrophage-lineage cells by regulating beta-oxidation-dependent mitochondrial ROS production. Cell Metab. 18, 265-278 (2013).

8. West, A. P. et al. TLR signalling augments macrophage bactericidal activity through mitochondrial ROS. Nature 472, 476-480 (2011)

9. Proksch, E., Brandner, J. M. \& Jensen, J.-M. The skin: an indispensable barrier. Exp. Dermatol. 17, 1063-1072 (2008).

10. Kupper, T. S. Immune and inflammatory processes in cutaneous tissues. Mechanisms and speculations. J. Clin. Invest. 86, 1783-1789 (1990).

11. Boguniewicz, M. \& Leung, D. Y. Atopic dermatitis: a disease of altered skin barrier and immune dysregulation. Immunol. Rev. 242, 233-246 (2011).

12. Pilcher, B. K. et al. Role of matrix metalloproteinases and their inhibition in cutaneous wound healing and allergic contact hypersensitivity. Ann. NY Acad. Sci. 878, 12-24 (1999).

13. Purwar, R., Kraus, M., Werfel, T. \& Wittmann, M. Modulation of keratinocytederived MMP-9 by IL-13: a possible role for the pathogenesis of epidermal inflammation. J. Invest. Dermatol. 128, 59-66 (2008).

14. Nelson, K. K. \& Melendez, J. A. Mitochondrial redox control of matrix metalloproteinases. Free Radic. Biol. Med. 37, 768-784 (2004).

15. Song, H. Y. et al. Suppression of TNF-alpha-induced MMP-9 expression by a cell-permeable superoxide dismutase in keratinocytes. BMB Rep. 44, 462-467 (2011).

16. Trede, N. S., Langenau, D. M., Traver, D., Look, A. T. \& Zon, L. I. The use of zebrafish to understand immunity. Immunity 20, 367-379 (2004).

17. Ellett, F., Pase, L., Hayman, J. W., Andrianopoulos, A. \& Lieschke, G. J. mpeg1 promoter transgenes direct macrophage-lineage expression in zebrafish. Blood 117, e49-e56 (2011)
18. Hall, C. et al. Transgenic zebrafish reporter lines reveal conserved Toll-like receptor signaling potential in embryonic myeloid leukocytes and adult immune cell lineages. J. Leukoc. Biol. 85, 751-765 (2009).

19. Hall, C., Flores, M. V., Storm, T., Crosier, K. \& Crosier, P. The zebrafish lysozyme $\mathrm{C}$ promoter drives myeloid-specific expression in transgenic fish. BMC. Dev. Biol. 7, 42 (2007).

20. Renshaw, S. A. et al. A transgenic zebrafish model of neutrophilic inflammation. Blood 108, 3976-3978 (2006).

21. Mathias, J. R. et al. Resolution of inflammation by retrograde chemotaxis of neutrophils in transgenic zebrafish. J. Leukoc. Biol. 80, 1281-1288 (2006).

22. Mathias, J. R. et al. Live imaging of chronic inflammation caused by mutation of zebrafish Hail. J. Cell Sci. 120, 3372-3383 (2007).

23. Niethammer, P., Grabher, C., Look, A. T. \& Mitchison, T. J. A tissue-scale gradient of hydrogen peroxide mediates rapid wound detection in zebrafish. Nature 459, 996-999 (2009).

24. Yoo, S. K., Starnes, T. W., Deng, Q. \& Huttenlocher, A. Lyn is a redox sensor that mediates leukocyte wound attraction in vivo. Nature 480, 109-112 (2011).

25. Le Guellec, D., Morvan-Dubois, G. \& Sire, J. Y. Skin development in bony fish with particular emphasis on collagen deposition in the dermis of the zebrafish (Danio rerio). Int. J. Dev. Biol. 48, 217-231 (2004).

26. Chang, W. J. \& Hwang, P. P. Development of zebrafish epidermis. Birth Defects Res. C Embryo Today 93, 205-214 (2011).

27. Li, Q. \& Uitto, J. Zebrafish as a model system to study heritable skin diseases. Methods Mol. Biol. 961, 411-424 (2013).

28. Li, Q., Frank, M., Thisse, C. I., Thisse, B. V. \& Uitto, J. Zebrafish: a model system to study heritable skin diseases. J. Invest. Dermatol. 131, 565-571 (2011).

29. Chen, B., Zhang, D. \& Pollard, J. W. Progesterone regulation of the mammalian ortholog of methylcitrate dehydratase (immune response gene 1 ) in the uterine epithelium during implantation through the protein kinase $\mathrm{C}$ pathway. Mol. Endocrinol. 17, 2340-2354 (2003).

30. van Soest, J. J. et al. Comparison of static immersion and intravenous injection systems for exposure of zebrafish embryos to the natural pathogen Edwardsiella tarda. BMC Immunol. 12, 58 (2011).

31. Hall, C. J. et al. Infection-responsive expansion of the hematopoietic stem and progenitor cell compartment in zebrafish is dependent upon inducible nitric oxide. Cell Stem Cell 10, 198-209 (2012).

32. Su, F. et al. Differential regulation of primitive myelopoiesis in the zebrafish by Spi-1/Pu.1 and C/ebp1. Zebrafish 4, 187-199 (2007).

33. Li, L., Jin, H., Xu, J., Shi, Y. \& Wen, Z. Irf8 regulates macrophage versus neutrophil fate during zebrafish primitive myelopoiesis. Blood 117, 1359-1369 (2011).

34. Trinchieri, G. \& Sher, A. Cooperation of Toll-like receptor signals in innate immune defence. Nat. Rev. Immunol. 7, 179-190 (2007).

35. van der Sar, A. M. et al. MyD88 innate immune function in a zebrafish embryo infection model. Infect. Immun. 74, 2436-2441 (2006).

36. Zhang, Y. et al. In vivo interstitial migration of primitive macrophages mediated by JNK-matrix metalloproteinase 13 signaling in response to acute injury. J. Immunol. 181, 2155-2164 (2008).

37. Liu, Y. W. Interrenal organogenesis in the zebrafish model. Organogenesis 3, $44-48$ (2007)

38. Van Lint, P. \& Libert, C. Chemokine and cytokine processing by matrix metalloproteinases and its effect on leukocyte migration and inflammation. J. Leukoc. Biol. 82, 1375-1381 (2007).

39. Volkman, H. E. et al. Tuberculous granuloma induction via interaction of a bacterial secreted protein with host epithelium. Science 327, 466-469 (2010).

40. Gray, C. et al. Simultaneous intravital imaging of macrophage and neutrophil behaviour during inflammation using a novel transgenic zebrafish. Thromb. Haemost. 105, 811-819 (2011)

41. Pase, L. et al. Neutrophil-delivered myeloperoxidase dampens the hydrogen peroxide burst after tissue wounding in zebrafish. Curr. Biol. 22, 1818-1824 (2012).

42. Bai, S. et al. Matrix metalloproteinase expression and function during fin regeneration in zebrafish: analysis of MT1-MMP, MMP2 and TIMP2. Matrix Biol. 24, 247-260 (2005).

43. Harper, J. I. et al. A study of matrix metalloproteinase expression and activity in atopic dermatitis using a novel skin wash sampling assay for functional biomarker analysis. Br. J. Dermatol. 162, 397-403 (2010).

44. Li, H. et al. Different neurotropic pathogens elicit neurotoxic CCR9- or neurosupportive CXCR3-expressing microglia. J. Immunol. 177, 3644-3656 (2006).

45. Degrandi, D., Hoffmann, R., Beuter-Gunia, C. \& Pfeffer, K. The proinflammatory cytokine-induced IRG1 protein associates with mitochondria J. Interferon Cytokine Res. 29, 55-67 (2009).

46. Lee, C. G., Jenkins, N. A., Gilbert, D. J., Copeland, N. G. \& O’Brien, W. E. Cloning and analysis of gene regulation of a novel LPS-inducible cDNA. Immunogenetics 41, 263-270 (1995). 
47. Sena, L. A. \& Chandel, N. S. Physiological roles of mitochondrial reactive oxygen species. Mol. Cell 48, 158-167 (2012).

48. Svineng, G., Ravuri, C., Rikardsen, O., Huseby, N. E. \& Winberg, J. O. The role of reactive oxygen species in integrin and matrix metalloproteinase expression and function. Connect. Tissue Res. 49, 197-202 (2008).

49. Vickers, A. E. Characterization of hepatic mitochondrial injury induced by fatty acid oxidation inhibitors. Toxicol. Pathol. 37, 78-88 (2009).

50. Barber, P. A., Rushforth, D., Agrawal, S. \& Tuor, U. I. Infrared optical imaging of matrix metalloproteinases (MMPs) up regulation following ischemia reperfusion is ameliorated by hypothermia. BMC Neurosci. 13, 76 (2012).

51. Yoong, S. et al. Characterization of the zebrafish matrix metalloproteinase 9 gene and its developmental expression pattern. Gene. Expr. Patterns 7, 39-46 (2007).

52. Deng, Q. et al. Localized bacterial infection induces systemic activation of neutrophils through Cxcr2 signaling in zebrafish. J. Leukoc. Biol. 93, 761-769 (2013).

53. Cheon, Y. P., Xu, X., Bagchi, M. K. \& Bagchi, I. C. Immune-responsive gene 1 is a novel target of progesterone receptor and plays a critical role during implantation in the mouse. Endocrinology 144, 5623-5630 (2003).

54. Michelucci, A. et al. Immune-responsive gene 1 protein links metabolism to immunity by catalyzing itaconic acid production. Proc. Natl Acad. Sci. USA 110, 7820-7825 (2013).

55. Lodhi, I. J. \& Semenkovich, C. F. Peroxisomes: a nexus for lipid metabolism and cellular signaling. Cell Metab. 19, 380-392 (2014).

56. Horner, S. M., Liu, H. M., Park, H. S., Briley, J. \& Gale, Jr. M. Mitochondrialassociated endoplasmic reticulum membranes (MAM) form innate immune synapses and are targeted by hepatitis C virus. Proc. Natl Acad. Sci. USA 108, 14590-14595 (2011)

57. Postlethwait, J., Amores, A., Cresko, W., Singer, A. \& Yan, Y. L. Subfunction partitioning, the teleost radiation and the annotation of the human genome. Trends Genet. 20, 481-490 (2004).

58. Lynch, M. \& Conery, J. S. The evolutionary fate and consequences of duplicate genes. Science 290, 1151-1155 (2000).

59. Parks, W. C., Wilson, C. L. \& Lopez-Boado, Y. S. Matrix metalloproteinases as modulators of inflammation and innate immunity. Nat. Rev. Immunol. 4, 617-629 (2004).

60. Shin, M. H. et al. Reactive oxygen species produced by NADPH oxidase, xanthine oxidase, and mitochondrial electron transport system mediate heat shock-induced MMP-1 and MMP-9 expression. Free Radic. Biol. Med. 44, 635-645 (2008)

61. Madsen, P., Rasmussen, H. H., Leffers, H., Honore, B. \& Celis, J. E. Molecular cloning and expression of a novel keratinocyte protein (psoriasis-associated fatty acid-binding protein [PA-FABP]) that is highly up-regulated in psoriatic skin and that shares similarity to fatty acid-binding proteins. J. Invest. Dermatol. 99, 299-305 (1992).

62. Masouye, I., Saurat, J. H. \& Siegenthaler, G. Epidermal fatty-acid-binding protein in psoriasis, basal and squamous cell carcinomas: an immunohistological study. Dermatology 192, 208-213 (1996).

63. Ogawa, E. et al. Epidermal FABP (FABP5) regulates keratinocyte differentiation by $13(\mathrm{~S})$-HODE-mediated activation of the NF-kappaB signaling pathway. J. Invest. Dermatol. 131, 604-612 (2011).
64. Caspary, F. et al. A new therapeutic approach to treat psoriasis by inhibition of fatty acid oxidation by Etomoxir. Br. J. Dermatol. 153, 937-944 (2005).

65. Jowett, T. \& Lettice, L. Whole-mount in situ hybridizations on zebrafish embryos using a mixture of digoxigenin- and fluorescein-labelled probes. Trends Genet. 10, 73-74 (1994).

66. Rhodes, J. et al. Interplay of pu.1 and gatal determines myelo-erythroid progenitor cell fate in zebrafish. Dev. Cell 8, 97-108 (2005).

67. Hall, C., Flores, M. V., Crosier, K. \& Crosier, P. Live cell imaging of zebrafish leukocytes. Methods Mol. Biol. 546, 255-271 (2009).

68. Oehlers, S. H. et al. A chemical enterocolitis model in zebrafish larvae that is dependent on microbiota and responsive to pharmacological agents. Dev. Dyn. 240, 288-298 (2011).

69. Oehlers, S. H. et al. The inflammatory bowel disease (IBD) susceptibility genes NOD1 and NOD2 have conserved anti-bacterial roles in zebrafish. Dis. Model Mech. 4, 832-841 (2011).

70. Sun, X., Zhang, J., Macgibbon, A. K., Black, P. \& Krissansen, G. W. Bovine milk fat enriched in conjugated linoleic and vaccenic acids attenuates allergic dermatitis in mice. Clin. Exp. Allergy 41, 729-738 (2011).

\section{Acknowledgements}

We thank Annie Chien and Alisha Malik for excellent technical assistance and Alhad Mahagaonkar for expert management of our zebrafish facility. We thank the Biomedical Imaging Research Unit, School of Medical Sciences, University of Auckland, for expert assistance in fluorescence and confocal microscopy and Stephen Edgar for flow cytometry support. We also thank Alan Davidson for supplying the cyp11a1 probe template and Graham Lieschke for providing the mpegl transgenic reporter lines. Funding for this work was provided by a grant awarded to P.S.C from the Ministry of Business, Innovation and Employment, New Zealand.

\section{Author contributions}

C.J.H., R.H.B., X.S., S.M.W., J.P.M, G.W.K and C.G.P performed experiments and helped analyse the data. C.J.H., K.E.C and P.S.C. wrote the manuscript. All authors approved of the final version.

\section{Additional information}

Accession codes: Microarray data used in this study have been deposited in the Gene Expression Omnibus, accession number GSE56365.

Supplementary Information accompanies this paper at http://www.nature.com/ naturecommunications

Competing financial interests: The authors declare no competing financial interests

Reprints and permission information is available online at http://npg.nature.com/ reprintsandpermissions/

How to cite this article: Hall, C. J. et al. Epidermal cells help coordinate leukocyte migration during inflammation through fatty acid-fuelled matrix metalloproteinase production. Nat. Commun. 5:3880 doi: 10.1038/ncomms4880 (2014). 